\title{
Multicriteria q-Rung Orthopair Fuzzy Decision Analysis: A Novel Approach Based on Archimedean Aggregation Operators with the Confidence Levels
}

Yabin Shao ( $\nabla$ shaoyb@cqupt.edu.cn )

Chongqing University of Posts and Telecommunications https://orcid.org/0000-0003-1001-7132

\section{Ning Wang}

Chongqing University of Posts and Telecommunications

\section{Zengtai Gong}

Northwest Normal University

\section{Research Article}

Keywords: Nonstandard fuzzy sets, Confidence q-rung orthopair fuzzy sets, Archimedean t-norm and tconorm, Aggregation operator, Multicriteria group decision-making

Posted Date: January 10th, 2022

DOI: https://doi.org/10.21203/rs.3.rs-1059359/v1

License: (a) (1) This work is licensed under a Creative Commons Attribution 4.0 International License. Read Full License

Version of Record: A version of this preprint was published at Soft Computing on February 5th, 2022. See the published version at https://doi.org/10.1007/s00500-022-06776-8. 


\title{
Multicriteria q-Rung Orthopair Fuzzy Decision Analysis: A Novel Approach Based on Archimedean Aggregation Operators with the Confidence Levels
}

\author{
Yabin Shao* · Ning Wang · Zengtai Gong
}

Received: date / Accepted: date

\begin{abstract}
The confidence levels can reduce the influence of the unreasonable evaluation value was given by the decision maker on the decision-making results. The Archimedean t-norm and t-conorm (ATS) also have many advantages for the processing of uncertain data. Under this environment, the confidence q-rung orthopair fuzzy aggregation operators based on ATS is one of the most successful extensions of confidence q-rung orthopair fuzzy numbers (Cq-ROFNs) in which decrease the deviation caused by the subjective perspective of the decision maker in the multicriteria group decision-making (MCGDM) problems. In this paper, we propose weighted, ordered weighted averaging aggregation operators and weighted, ordered weighted geometric aggregation operators based on ATS, respectively. Moreover, the properties and four specific forms associated with aggregation operators are also investigated. In this study, a novel MCGDM approach is introduced by using the proposed operator. A reasonable example is proposed and compared the results which are obtained by our operators and that in existing literature, so as to verify the rationality and flexible of our method. From the study, we concluded that the proposed method can reduce the impact of extreme data, and makes decision-making results more reasonable by considering the attitudes of decision-makers.
\end{abstract}

Yabin Shao*, Corresponding author.

School of Science, Chongqing University of Posts and Telecommunications, Chongqing 400065, P. R. China

Tel.: +86-23-6276-0289

E-mail: shaoyb@cqupt.edu.cn

Ning Wang

School of Science, Chongqing University of Posts and Telecommunications, Chongqing 400065, P. R. China

E-mail: s180602015@stu.cqupt.edu.cn

Zengtai Gong

College of Mathematics and Statistics, Northwest Normal University, Lanzhou 730070, P. R. China

E-mail: zt-gong@163.com 
Keywords Nonstandard fuzzy sets · Confidence q-rung orthopair fuzzy sets . Archimedean t-norm and t-conorm - Aggregation operator · Multicriteria group decision-making

\section{Introduction}

In recent years, due to the complexity of objective things, uncertainty and the fuzziness of cognitive-based human thinking, the research on multi-attribute decision making (MCGDM) problems in uncertain environment has attracted great attention of scholars. The MCGDM is generally a process in which the decision makers concentrate on selecting the best option among all the alternatives to be selected and some related theories are presented $[38,20,3,16]$. Its prominent advantage is that multi-person decision-making reflects the fairness and rationality, which can greatly reduces the influence of individual decision-making by its own professional backgrounds and subjective factors. For the real decision-making process, the pivotal problem is whether the evaluating value can be expressed reasonably. However, because the decision-making body is generally human, the evaluation value is usually uncertain and vague, which requires a reasonable tool to express it. On the other hand, most extant classical mathematical theory is used to process accurate data, whereas the fuzziness of decision data leads to the inability of traditional mathematical tools to meet the demand. For this, Zadeh [49] extended the classical sets of the characteristic functions with value of 0 or 1 , and presented fuzzy sets (FSs) theory whose membership function is within $[0,1]$. However, we have to consider the degree of hesitancy on some issues, such as the voting model. In order to solve this problem, Atanassov [1,2] proposed intuitionistic fuzzy sets (IFSs), which have investigated from three aspects: membership degree (MD), nonmembership degree (NMD) and hesitancy degree (HD). Therefore, the theory of IFSs has more practicality and comprehensiveness when it is portraying uncertainty models. Based on which, many researches with regard to IFSs have emerged and applied in medical diagnosis [35], pattern recognition [6] and group decision-making (GDM) [18]. These studies mainly carried out from five different fields.

1. The basic method research: For instance, operation laws [5], entropy measure [4], fuzzy measure [37], divengence measure [14], distance and similarity measure [6, 35], etc.

2. The extended MCGDM methods: Such as Vlsekriterijumska Optimizacija I Kompromisno Resenje(VIKOR) method [40], Dampster-Shafter theory(DST) [26], an acronym in Portuguese of interactive and multi-criteria decision-making(TODIM) [30], etc.

3. The extended aggregation operators(AOs): It is well known that the AOs can effectively aggregate information, some related methods have been investigated. For example, $\mathrm{Xu}$ [41] first proposed several intuitionistic fuzzy AOs. $\mathrm{Xu}$ and Yager [42] presented some intuitionistic fuzzy geometric AOs.

4. Combining the IFSs with other methods: For example, intuitionistic fuzzy rough sets [50-52], Interval valued intuitionistic uncertain linguistic variables [18], hesitantintuitionistic fuzzy information [53] etc. 
5. The extended evaluating value range: For example, interval valued IFSs [2], trapezoidal intuitionistic fuzzy numbers(IFNs) [40], triangular IFNs [40,30], etc.

Nevertheless, it is noted that $\operatorname{MD}\left(\mu_{Q}\right)$ and $\operatorname{NMD}\left(v_{Q}\right)$ satisfy $\mu_{Q}+v_{Q}>1$ under certain conditions. For example, a expert provide an evaluation value with MD is 0.9 while NMD is 0.4 , this severely exceeds the range of application of IFSs. To handle it, Yager [45] presented Pythagorean fuzzy sets (PFSs) whose remarkable characteristic is that MD and NMD satisfy $\mu_{Q}^{2}+v_{Q}^{2} \leq 1$. Therefore, Yager successfully extended fuzzy theory so that it can more accurately and reasonably denote uncertainty information. Hereafter, some research results of PFSs are gradually commence. For instance, Yager [46] defined several AOs based on Pythagorean fuzzy enviroment. Garg [9], [10] presented some generalized AOs based on Einstein operations in the Pythagorean fuzzy environment and applied to realistic MCDM problems. Garg [7] proposed a novel Pythagorean fuzzy geometric AOs based on neutral multiplication and power operational rules by considering neutrally treat the MD and NMD.

As the MCGDM problem becomes more complex, Yager [47] proposed q-rung orthopair fuzzy sets (q-ROFSs), which $\mu_{Q}, v_{Q}$ satisfies $\mu_{Q}^{q}+v_{Q}^{q} \leq 1$. The q-ROFSs can be regarded as the extension and supplement of the IFSs and the PFSs, so it can convey and handle more uncertain information. After that, many scholars have done a lot of research with respect to related operations and applications based on q-ROFSs. For example, Liu and Wang [21] showed that q-rung orthopair fuzzy information AOs and their applications on MCDM, such as q-rung orthopair fuzzy weighted averaging (qROFWA) operator, the q-rung orthopair fuzzy weighted geometric (q-ROFWG) operator. Joshi et al. [13] proposed interval valued q-rung orthopair fuzzy sets and their complement operation and aggregation. Liu et al. [19] proposed q-rung orthopair fuzzy partitioned Heronian mean operators (q-ROFPHM) to solve MCGDM problems, which considers the division of similar attributes in all attributes into one class, further optimizing the interaction between different attributes.

We usually use AOs and other extensions MCGDM theory such as DST, TODIM to solve the problem. However, the difference between these two types is that AOs can get the sorting results and specific values, whereas the extended MCGDM only gets the sorting results. Therefore, the AOs can aggregate information more comprehensively. The study of AOs can be divided into operational law and aggregation function, where operational laws have Archimedean t-norm and t-conorm (ATS) and its special cases, aggregation function have Heronian mean [19], Bonferroni mean [24], etc.

In the decision-making problems, the evaluation value was given by the expert should first be ensured to be fair and reasonable, and then the results obtained by data fusion should not be too inaccurate. However, the above AOs do not take into account the different confidence levels of each expert on the attributes of the evaluation object. To overcome this shortcoming, Xia et al. [43] proposed a series of confidence induced weighted aggregation operators in the context of fuzzy sets and hesitant fuzzy sets. Yu [48] further studied confidence intuitionistic fuzzy information AOs based on the algebraic operations and the Einstein operations, including the confidence intuitionistic fuzzy weighted averaging (CIFWA) operator, confidence intuitionistic fuzzy Einstein weighted geometric (CIFEWG) operator etc. After that, Garg [8] proposed confi- 
dence Pythagorean fuzzy information AOs. Based on the existing theories, Joshi and Gegov [15] proposed confidence q-rung orthopair fuzzy AOs, such as confidence qrung orthopair fuzzy weighted averaging (Cq-ROFWA) operator, confidence q-rung orthopair fuzzy weighted geometric (Cq-ROFWG) operator and so forth. For more applications, see [28,31-33] and the references therein.

As discussed above, we can find that the methods in the existing literature have both advantages and disadvantages, as following:

1. The confidence levels is an indispensable criterion to measure whether the evaluating value was given by experts is fair and reasonable. By changing its value, the influence of unreasonable or extreme data on the result can be balanced.

2. Introducing the confidence levels into various fuzzy AOs has significant advantages, which can reduce the influence of decision makers' subjective factors.

3. Although the confidence levels has been considered in the fuzzy decision-making, it is not sufficient to solve most decision-making problems based on certain kinds of operational rules. The ATS is a more generalized form of several operational rules, which can provide more operational rules for fuzzy AOs and make them adapt to different decision environments.

According to the aforementioned analysis, in order to overcome these shortcomings, we have to developed some new methods to solve MCGDM problem more optimally. For this, we propose several novel AOs by combining the Cq-ROFNs into the ATS, and achieve the following goals.

1. The novel AOs can accommodate to various complex decision environments.

2. The novel AOs is easy to understand, calculate, and it has strong practical significance and application.

3. The proposed method can reduce the influence of extreme data and make it more reasonable for the decision results of practical problems.

In this paper, we present some confidence q-rung orthopair fuzzy information AOs based on ATS, including the confidence q-rung orthopair fuzzy weighted averaging operator based on ATS (ATS-Cq-ROFWA), the confidence q-rung orthopair fuzzy geometric operator based on ATS (ATS-Cq-ROFWG), the q-rung orthopair fuzzy ordered weighted averaging operator based on ATS (ATS-Cq-ROFOWA), and the confidence q-rung orthopair fuzzy ordered weighted geometric operator based on ATS (ATS-Cq-ROFOWG). Furthermore, we define a novel MCGDM method based on the proposed AOs. Finally, we illustrate an example to demonstrate the rationality and superiority of the proposed method, and we verify that the proposed method is superior to the existing literatures $[21,22]$ for solving real MCGDM problems.

The rest of this paper is composed of the following parts: In section II, some basic concepts and related operational laws of q-ROFSs and ATS are briefly introduced. In section III, we present some novel aggregation operators and discuss the properties associated with aggregation operators. Besides, we also give four special forms of each aggregation operator, such as the confidence q-rung orthopair fuzzy Hammer weighted averaging operator (Cq-ROFHWA), the confidence q-rung orthopair fuzzy Frank weighted geometric operator (Cq-ROFFWG) and so on. In section IV, we discuss a novel MCGDM method based on the proposed AOs. In section V, an example 
is provided to illustrate the feasibility and advantages of the novel approach. Then, by comparing with the existing integrate method, the correctness and advantages of the proposed method is verified. In section VI, we give some conclusion remarking and future research.

\section{Basic Definitions and Theorems}

In the following, we briefly give some relevant basic concepts.

Definition 1 [47] Let $X$ be a fixed set, a q-ROFS $Q$ in $X$ can be described as:

$$
Q=\left\{\left\langle x, \mu_{Q}(x), v_{Q}(x)\right\rangle \mid x \in X\right\}
$$

where $\mu_{Q}: X \rightarrow[0,1]$ represent the MD, $v_{Q}: X \rightarrow[0,1]$ represent the NMD, and $\mu_{Q}(x), v_{Q}(x)$ satisfy the condition of $\quad 0 \leq\left(\mu_{Q}(x)\right)^{q}+\left(v_{Q}(x)\right)^{q} \leq 1(q \geq 1)$. In addition, $\pi_{Q}(x)=\left(1-\left(\mu_{Q}(x)\right)^{q}-\left(v_{Q}(x)\right)^{q}\right)^{\frac{1}{q}}$ represent the degree of hesitancy, for all $x \in X$.

For convenience, $\phi=(\mu, v)$ is called q-rung orthopair fuzzy number (q-ROFN) by Yager [47].

In this section, we introduce score function proposed by Liu and Wang [21] to solve the MCGDM problem.

Definition 2 [21] If $\phi=(\mu, v)$ be a q-ROFN, then its score function is given by:

$$
S(\phi)=\mu^{q}-v^{q} .
$$

Definition 3 [21] If $\phi=(\mu, v)$ be a q-ROFN, then its accuracy function is given by:

$$
H(\phi)=\mu^{q}+v^{q} .
$$

Definition 4 [21] Let $\phi_{1}=\left(\mu_{1}, v_{1}\right)$ and $\phi_{2}=\left(\mu_{2}, v_{2}\right)$ be two q-ROFNs, then

1. If $S\left(\phi_{1}\right)>S\left(\phi_{2}\right)$, then $\phi_{1}>\phi_{2}$;

2. If $S\left(\phi_{1}\right)=S\left(\phi_{2}\right)$, then

(a) If $H\left(\phi_{1}\right)>H\left(\phi_{2}\right)$, then $\phi_{1}>\phi_{2}$;

(b) If $H\left(\phi_{1}\right)=H\left(\phi_{2}\right)$, then $\phi_{1}=\phi_{2}$;

Definition 5 [44] If a function $T:[0,1] \times[0,1] \rightarrow[0,1]$ satisfies the following conditions:

1. $T(1, x)=x$, for all $x$;

2. $T(x, y)=T(y, x)$, for all $x$ and $y$;

3. $T(x, T(y, z))=T(T(x, y), z)$, for all $x, y$ and $z$;

4. If $x \leq x^{\prime}$ and $y \leq y^{\prime}$,then $T(x, y) \leq T\left(x^{\prime}, y^{\prime}\right)$.

Then it is called a t-norm.

Definition 6 [44] If a function $S:[0,1] \times[0,1] \rightarrow[0,1]$ satisfies the following conditions: 
1. $S(0, x)=x$, for all $x$;

2. $S(x, y)=S(y, x)$, for all $x$ and $y$;

3. $S(x, S(y, z))=S(S(x, y), z)$, for all $x, y$ and $z$;

4. If $x \leq x^{\prime}$ and $y \leq y^{\prime}$,then $S(x, y) \leq S\left(x^{\prime}, y^{\prime}\right)$.

Then it is called a t-conorm.

Definition 7 [44] Suppose a t-norm function $T(x, y)$ is continuous and $T(x, x)<x$ for all $x \in[0,1]$, then it is called Archimedean t-norm(AT). If the AT is strictly decreasing in each variable for $x, y \in(0,1)$, it is called the strict AT.

Definition 8 [44] Suppose a t-conorm function $S(x, y)$ is continuous and $S(x, x)<x$ for all $x \in[0,1]$, then it is called AS. If the AS is strictly increasing in each variable for $x, y \in(0,1)$, it is called the strict AS.

According to [17],we can know that $T(x, y)$ is denoted as $T(x, y)=g^{-1}(g(x)+$ $g(y))$ by its additive generator $g$, and $S(x, y)$ is denoted as $S(x, y)=h^{-1}(h(x)+h(y))$ by its additive generator $h$, where $h(t)=g(1-t)$.

\section{3 q-Rung orthopair fuzzy information AOs with the confidence levels based on ATS}

For real MCGDM problems, we need not only the decision maker to give the evaluation value of the evaluation object, but also to give the confidence levels of the evaluation value. In this section, we propose the confidence q-rung orthopair fuzzy AOs based on ATS (ATS-Cq-ROFAOs) along with their special cases. In addition, we also put forward their corresponding properties.

\subsection{ATS-Cq-ROFWA operator}

Definition 9 Let $\alpha_{i}=\left(\mu_{i}, v_{i}\right)(i=1,2, \ldots, n)$ be a collection of q-ROFNs. The ATSCq-ROFWA operator is denoted as

$$
A T S-C q-R O F W A\left(\left\langle\lambda_{1}, \alpha_{1}\right\rangle,\left\langle\lambda_{2}, \alpha_{2}\right\rangle, \ldots,\left\langle\lambda_{n}, \alpha_{n}\right\rangle\right)=\bigoplus_{i=1}^{n} \omega_{i}\left(\lambda_{i} \alpha_{i}\right)
$$

where $\lambda_{i}$ and $\omega_{i}$ be the confidence levels and weight vector of $\alpha_{i}$, respectively, and taking $0 \leq \lambda_{i} \leq 1, \omega_{i} \in[0,1]$ and $\sum_{i=1}^{n} \omega_{i}=1$.

Remark 1 If $\lambda_{1}=\lambda_{2}=\ldots=\lambda_{n}=1$, then the ATS-Cq-ROFWA operator reduces to the q-rung orthopair fuzzy weighted averaging operator based on ATS(ATS-qROFWA)

$$
A T S-q-R O F W A\left(\left\langle\alpha_{1}\right\rangle,\left\langle\alpha_{2}\right\rangle, \ldots,\left\langle\alpha_{n}\right\rangle\right)=\bigoplus_{i=1}^{n} \omega_{i}\left(\alpha_{i}\right)
$$


Theorem 1 Let $\alpha_{i}=\left(\mu_{i}, v_{i}\right)(i=1,2, \ldots, n)$ be a collection of $q$-ROFNs and $\lambda_{i}$ and $\omega_{i}$ be the confidence levels and weight vector of $\alpha_{i}$, respectively. Then the aggregated value of $\alpha_{i}$ obtained by ATS-Cq-ROFWA operator is an q-ROFNs and

$$
\begin{aligned}
& A T S-C q-\text { ROFWA }\left(\left\langle\lambda_{1}, \alpha_{1}\right\rangle,\left\langle\lambda_{2}, \alpha_{2}\right\rangle, \ldots,\left\langle\lambda_{n}, \alpha_{n}\right\rangle\right) \\
& =\bigoplus_{i=1}^{n} \omega_{i}\left(\lambda_{i} \alpha_{i}\right)=\left(h^{-1}\left(\sum_{i=1}^{n} \omega_{i}\left(\lambda_{i} h\left(\mu_{i}\right)\right)\right), g^{-1}\left(\sum_{i=1}^{n} \omega_{i}\left(\lambda_{i} g\left(v_{i}\right)\right)\right)\right)
\end{aligned}
$$

where $0 \leq \lambda_{i} \leq 1, \sum_{i=1}^{n} \omega_{i}=1$ and $\omega_{i} \in(0,1)$.

Proof. We prove the Eq. (6) by mathematical induction.

(1) For $n=2$, that is

$$
\begin{aligned}
& A T S-C q-\operatorname{ROFWA}\left(\left\langle\lambda_{1}, \alpha_{1}\right\rangle,\left\langle\lambda_{2}, \alpha_{2}\right\rangle, \ldots,\left\langle\lambda_{n}, \alpha_{n}\right\rangle\right) \\
& =\bigoplus_{i=1}^{2} \omega_{i}\left(\lambda_{i} \alpha_{i}\right)=\omega_{1}\left(\lambda_{1} \alpha_{1}\right) \oplus \omega_{2}\left(\lambda_{2} \alpha_{2}\right) \\
& =\left(h^{-1}\left(h\left(h^{-1}\left(\omega_{1} h\left(h^{-1}\left(\lambda_{1} h\left(\mu_{1}\right)\right)\right)\right)\right)+h\left(h^{-1}\left(\omega_{2} h\left(h^{-1}\left(\lambda_{2} h\left(\mu_{2}\right)\right)\right)\right)\right)\right)\right. \text {, } \\
& \left.g^{-1}\left(g\left(g^{-1}\left(\omega_{1} g\left(g^{-1}\left(\lambda_{1} g\left(v_{1}\right)\right)\right)\right)\right)+g\left(g^{-1}\left(\omega_{2} g\left(g^{-1}\left(\lambda_{2} g\left(v_{2}\right)\right)\right)\right)\right)\right)\right) \\
& =\left(h^{-1}\left(\omega_{1} \lambda_{1} h\left(\mu_{1}\right)+\omega_{2} \lambda_{2} h\left(\mu_{2}\right)\right), g^{-1}\left(\omega_{1} \lambda_{1} g\left(v_{1}\right)+\omega_{2} \lambda_{2} g\left(v_{2}\right)\right)\right) \text {. }
\end{aligned}
$$

(2) Given Eq. (6) holds for $n=k$, we can get

$$
\begin{aligned}
& \text { ATS-Cq-ROFWA }\left(\left\langle\lambda_{1}, \alpha_{1}\right\rangle,\left\langle\lambda_{2}, \alpha_{2}\right\rangle, \ldots,\left\langle\lambda_{n}, \alpha_{n}\right\rangle\right) \\
& =\bigoplus_{i=1}^{k} \omega_{i}\left(\lambda_{i} \alpha_{i}\right)=\left(h^{-1}\left(\sum_{i=1}^{k} \omega_{i} \lambda_{i} h\left(\mu_{i}\right)\right), g^{-1}\left(\sum_{i=1}^{k} \omega_{i} \lambda_{i} g\left(v_{i}\right)\right)\right) .
\end{aligned}
$$


8

Y. Shao, N. Wang and Z. Gong

$$
\begin{aligned}
& \text { (3) When } n=k+1 \text {, we have } \\
& A T S-C q-\operatorname{ROFWA}\left(\left\langle\lambda_{1}, \alpha_{1}\right\rangle,\left\langle\lambda_{2}, \alpha_{2}\right\rangle, \ldots,\left\langle\lambda_{n}, \alpha_{n}\right\rangle\right) \\
& =\bigoplus_{i=1}^{k} \omega_{i}\left(\lambda_{i} \alpha_{i}\right) \oplus \omega_{k+1}\left(\lambda_{k+1} \alpha_{k+1}\right) \\
& =\left(h^{-1}\left(\sum_{i=1}^{k} \omega_{i} \lambda_{i} h\left(\mu_{i}\right)\right), g^{-1}\left(\sum_{i=1}^{k} \omega_{i} \lambda_{i} g\left(v_{i}\right)\right)\right) \oplus \\
& \left(h^{-1}\left(\omega_{k+1} \lambda_{k+1} h\left(\mu_{k+1}\right)\right), g^{-1}\left(\omega_{k+1} \lambda_{k+1} g\left(v_{k+1}\right)\right)\right) \\
& =\left(h^{-1}\left(h\left(h^{-1}\left(\sum_{i=1}^{k} \omega_{i} \lambda_{i} h\left(\mu_{i}\right)\right)\right)+h\left(h^{-1}\left(\omega_{k+1} \lambda_{k+1} h\left(\mu_{k+1}\right)\right)\right)\right)\right. \text {, } \\
& \left.g^{-1}\left(g\left(g^{-1}\left(\sum_{i=1}^{k} \omega_{i} \lambda_{i} g\left(v_{i}\right)\right)\right)+g\left(g^{-1}\left(\omega_{k+1} \lambda_{k+1} g\left(v_{k+1}\right)\right)\right)\right)\right) \\
& =\left(h^{-1}\left(\sum_{i=1}^{k} \omega_{i} \lambda_{i} h\left(\mu_{i}\right)+\omega_{k+1} \lambda_{k+1} h\left(\mu_{k+1}\right)\right)\right. \text {, } \\
& \left.g^{-1}\left(\sum_{i=1}^{k} \omega_{i} \lambda_{i} g\left(v_{i}\right)+\omega_{k+1} \lambda_{k+1} g\left(v_{k+1}\right)\right)\right) \\
& =\left(h^{-1}\left(\sum_{i=1}^{k+1} \omega_{i} \lambda_{i} h\left(\mu_{i}\right)\right), g^{-1}\left(\sum_{i=1}^{k+1} \omega_{i} \lambda_{i} g\left(v_{i}\right)\right)\right) .
\end{aligned}
$$

That is Eq. (6) holds for $n=k+1$.

Now, we prove ATS-Cq-ROFWA operator is an q-ROFNs.

As can be seen from above, AT function $g(t):[0,1] \rightarrow[0, \infty]$ is strictly decreasing, and AS function $h(t)$ is strictly increasing, simultaneously, $h(t)$ and $g(t)$ are satisfied $h(t)=g(1-t)$, hence

$$
0 \leq h^{-1}\left(\sum_{i=1}^{n} \omega_{i}\left(\lambda_{i} h\left(\mu_{i}\right)\right)\right), g^{-1}\left(\sum_{i=1}^{n} \omega_{i}\left(\lambda_{i} g\left(v_{i}\right)\right)\right) \leq 1
$$

and

$$
\begin{aligned}
& h^{-1}\left(\sum_{i=1}^{n} \omega_{i}\left(\lambda_{i} h\left(\mu_{i}\right)\right)\right)+g^{-1}\left(\sum_{i=1}^{n} \omega_{i}\left(\lambda_{i} g\left(v_{i}\right)\right)\right) \\
& \leq h^{-1}\left(\sum_{i=1}^{n} \omega_{i}\left(\lambda_{i} h\left(\mu_{i}\right)\right)\right)+g^{-1}\left(\sum_{i=1}^{n} \omega_{i}\left(\lambda_{i} g\left(1-\mu_{i}\right)\right)\right) \\
& =h^{-1}\left(\sum_{i=1}^{n} \omega_{i}\left(\lambda_{i} h\left(\mu_{i}\right)\right)\right)+1-h^{-1}\left(\sum_{i=1}^{n} \omega_{i}\left(\lambda_{i} h\left(\mu_{i}\right)\right)\right) \\
& =1 .
\end{aligned}
$$

Therefore, this proof is completed.

Next, we present some basic properties of the ATS-Cq-ROFWA operator. 
Theorem 2 (Idempotency) Let $\alpha_{i}=\left(\mu_{i}, v_{i}\right)$ be a collection of $q$-ROFNs and suppose $\left(\alpha, \alpha_{1}, \alpha_{2}, \ldots, \alpha_{n}\right)$ are equal, which is $\alpha=\alpha_{i}=(\mu, v)$, for $i=1,2, \ldots, n$, then

$$
\text { ATS-Cq-ROFWA }\left(\left\langle\lambda_{1}, \alpha_{1}\right\rangle,\left\langle\lambda_{2}, \alpha_{2}\right\rangle, \ldots,\left\langle\lambda_{n}, \alpha_{n}\right\rangle\right)=\lambda \alpha .
$$

Proof. when $\alpha=\alpha_{1}=\alpha_{2}=\ldots=\alpha_{n}=(\mu, v)$, we can get

$$
\begin{aligned}
& \text { ATS-Cq-ROFWA }\left(\left\langle\lambda_{1}, \alpha_{1}\right\rangle,\left\langle\lambda_{2}, \alpha_{2}\right\rangle, \ldots,\left\langle\lambda_{n}, \alpha_{n}\right\rangle\right) \\
& =\operatorname{ATS}-C q-\operatorname{ROFWA}\left(\left\langle\lambda_{1}, \alpha\right\rangle,\left\langle\lambda_{2}, \alpha\right\rangle, \ldots,\left\langle\lambda_{n}, \alpha\right\rangle\right) \\
& =\bigoplus_{i=1}^{n} \omega_{i}\left(\lambda_{i} \alpha\right)=\left(h^{-1}\left(\sum_{i=1}^{n} \omega_{i} \lambda_{i} h(\mu)\right), g^{-1}\left(\sum_{i=1}^{n} \omega_{i} \lambda_{i} g(v)\right)\right) \\
& =\left(h^{-1}(\lambda h(\mu)), g^{-1}(\lambda g(v))\right)=\lambda \alpha .
\end{aligned}
$$

Theorem 3 (Monotonicity) Suppose $\alpha_{i}=\left(\alpha_{1}, \alpha_{2}, \ldots, \alpha_{n}\right), \ddot{\alpha}_{i}=\left(\ddot{\alpha}_{1}, \ddot{\alpha}_{2}, \ldots, \ddot{\alpha}_{n}\right)$ be two collections of $q$-ROFNs, when $\mu_{i} \leq \ddot{\mu}_{i}$ and $v_{i} \geq \ddot{v}_{i}$, we have

$$
\begin{aligned}
& \text { ATS-Cq-ROFWA }\left(\left\langle\lambda_{1}, \alpha_{1}\right\rangle,\left\langle\lambda_{2}, \alpha_{2}\right\rangle, \ldots,\left\langle\lambda_{n}, \alpha_{n}\right\rangle\right) \\
& \leq A T S-C q-\operatorname{ROFWA}\left(\left\langle\lambda_{1}, \ddot{\alpha}_{1}\right\rangle,\left\langle\lambda_{2}, \ddot{\alpha}_{2}\right\rangle, \ldots,\left\langle\lambda_{n}, \ddot{\alpha}_{n}\right\rangle\right) .
\end{aligned}
$$

Proof. We have learnt that $h(t)$ and $g(t)$ are strictly increasing function and strictly decreasing function, respectively. Since $\mu_{i} \leq \ddot{\mu}_{i}$ and $v_{i} \geq \ddot{v}_{i}$, then we have

$$
\begin{aligned}
& h^{-1}\left(\sum_{i=1}^{n} \omega_{i}\left(\lambda_{i} h\left(\mu_{i}\right)\right)\right) \leq h^{-1}\left(\sum_{i=1}^{n} \omega_{i}\left(\lambda_{i} h\left(\ddot{\mu}_{i}\right)\right)\right) \\
& g^{-1}\left(\sum_{i=1}^{n} \omega_{i}\left(\lambda_{i} g\left(v_{i}\right)\right)\right) \geq g^{-1}\left(\sum_{i=1}^{n} \omega_{i}\left(\lambda_{i} g\left(\ddot{v}_{i}\right)\right)\right) .
\end{aligned}
$$

Therefore

$$
\begin{aligned}
& \text { ATS-Cq-ROFWA }\left(\left\langle\lambda_{1}, \alpha_{1}\right\rangle,\left\langle\lambda_{2}, \alpha_{2}\right\rangle, \ldots,\left\langle\lambda_{n}, \alpha_{n}\right\rangle\right) \\
& \leq A T S-C q-R O F W A\left(\left\langle\lambda_{1}, \ddot{\alpha}_{1}\right\rangle,\left\langle\lambda_{2}, \ddot{\alpha}_{2}\right\rangle, \ldots,\left\langle\lambda_{n}, \ddot{\alpha}_{n}\right\rangle\right) .
\end{aligned}
$$

Theorem 4 (Boundedness) Suppose $\ddot{\alpha}_{i}=\left(\ddot{\alpha}_{1}, \ddot{\alpha}_{2}, \ldots, \ddot{\alpha}_{n}\right)$ be a collection of $q$-ROFNs, $\ddot{\alpha}_{\min }=\left(\min _{i}\left\{\lambda_{i} \ddot{\mu}_{i}\right\}, \max _{i}\left\{\lambda_{i} \ddot{v}_{i}\right\}\right)$, and $\ddot{\alpha}_{\max }=\left(\max _{i}\left\{\lambda_{i} \ddot{\mu}_{i}\right\}, \min _{i}\left\{\lambda_{i} \ddot{v}_{i}\right\}\right)$, then we have

$$
\ddot{\alpha}_{\text {min }} \leq A T S-C q-R O F W A\left(\left\langle\lambda_{1}, \ddot{\alpha}_{1}\right\rangle,\left\langle\lambda_{2}, \ddot{\alpha}_{2}\right\rangle, \ldots,\left\langle\lambda_{n}, \ddot{\alpha}_{n}\right\rangle\right) \leq \ddot{\alpha}_{\max } .
$$

Proof. The proof is similar to Theorem 3, consequently, it is omitted.

In the following, we give some series of special AOs for different additive generator $g(t)$.

(1) If $g(t)=-\log \left(t^{q}\right)$, we can get confidence q-rung orthopair fuzzy algebraic weighted averaging (Cq-ROFWA)[15] operator, that is

$$
\begin{aligned}
& \text { Cq-ROFWA }\left(\left\langle\lambda_{1}, \alpha_{1}\right\rangle,\left\langle\lambda_{2}, \alpha_{2}\right\rangle, \ldots,\left\langle\lambda_{n}, \alpha_{n}\right\rangle\right) \\
& =\left\langle\left(1-\prod_{i=1}^{n}\left(1-\mu_{i}^{q}\right)^{\lambda_{i} \omega_{i}}\right)^{\frac{1}{q}}, \prod_{i=1}^{n}\left(v_{i}\right)^{\lambda_{i} \omega_{i}}\right\rangle .
\end{aligned}
$$


(2) If $g(t)=\log \left(\frac{2-t^{q}}{t^{q}}\right)$, we can get confidence q-rung orthopair fuzzy Einstein weighted averaging (Cq-ROFEWA) operator, that is

$$
\begin{aligned}
& \operatorname{Cq}-\operatorname{ROFEWA}\left(\left\langle\lambda_{1}, \alpha_{1}\right\rangle,\left\langle\lambda_{2}, \alpha_{2}\right\rangle, \ldots,\left\langle\lambda_{n}, \alpha_{n}\right\rangle\right) \\
= & \left\langle\left(\frac{\prod_{i=1}^{n}\left(1+\mu_{i}^{q}\right)^{\lambda_{i} \omega_{i}}-\left(1-\mu_{i}^{q}\right)^{\lambda_{i} \omega_{i}}}{\prod_{i=1}^{n}\left(1+\mu_{i}^{q}\right)^{\lambda_{i} \omega_{i}}+\left(1-\mu_{i}^{q}\right)^{\lambda_{i} \omega_{i}}}\right)^{\frac{1}{q}},\left(\frac{2 \prod_{i=1}^{n}\left(v_{i}^{q}\right)^{\lambda_{i} \omega_{i}}}{\prod_{i=1}^{n}\left(2-v_{i}^{q}\right)^{\lambda_{i} \omega_{i}}+\prod_{i=1}^{n}\left(v_{i}^{q}\right)^{\lambda_{i} \omega_{i}}}\right)^{\frac{1}{q}}\right\rangle .
\end{aligned}
$$

(3) If $g(t)=\log \left(\frac{\rho+(1-\rho) t^{q}}{t^{q}}\right)$, we can get confidence q-rung orthopair fuzzy Hammer weighted averaging (Cq-ROFHWA) operator, that is

$$
\begin{aligned}
& \operatorname{Cq}-\operatorname{ROFHWA}\left(\left\langle\lambda_{1}, \alpha_{1}\right\rangle,\left\langle\lambda_{2}, \alpha_{2}\right\rangle, \ldots,\left\langle\lambda_{n}, \alpha_{n}\right\rangle\right) \\
= & \left\langle\left(\frac{\prod_{i=1}^{n}(a)^{\lambda_{i} \omega_{i}}-\prod_{i=1}^{n}(b)^{\lambda_{i} \omega_{i}}}{\prod_{i=1}^{n}(a)^{\lambda_{i} \omega_{i}}+(\rho-1) \prod_{i=1}^{n}(b)^{\lambda_{i} \omega_{i}}}\right)^{\frac{1}{q}},\left(\frac{\rho \prod_{i=1}^{n}(c)^{\lambda_{i} \omega_{i}}}{\prod_{i=1}^{n}(d)^{\lambda_{i} \omega_{i}}+(\rho-1) \prod_{i=1}^{n}(c)^{\lambda_{i} \omega_{i}}}\right)^{\frac{1}{q}}\right\rangle
\end{aligned}
$$

where $a=1+(\rho-1) \mu_{i}^{q}, b=1-\mu_{i}^{q}, c=v_{i}^{q}, d=1+(\rho-1)\left(1-v_{i}^{q}\right)$.

Remark 2 1. If $\rho=1$, then the Cq-ROFHWA operator reduces to the Cq-ROFWA operator [15];

2. If $\rho=2$, then the Cq-ROFHWA operator reduces to the Cq-ROFEWA operator;

3. If $\rho=1$ and $\lambda_{i}=1$, then the Cq-ROFHWA operator reduces to the q-ROFWA operator [21];

4. If $q=2, \rho=2$ and $\lambda_{i}=1$, then the Cq-ROFHWA operator reduces to the PFEWA operator [9].

(4) If $g(t)=\log \left(\frac{\rho-1}{\rho^{t^{q}}-1}\right), \rho>1$, we can get confidence q-rung orthopair fuzzy Frank weighted averaging (Cq-ROFFWA) operator, that is

$$
\begin{gathered}
C q-\operatorname{ROFFWA}\left(\left\langle\lambda_{1}, \alpha_{1}\right\rangle,\left\langle\lambda_{2}, \alpha_{2}\right\rangle, \ldots,\left\langle\lambda_{n}, \alpha_{n}\right\rangle\right) \\
=\left\langle\left(1-\log _{\rho}\left(1+\frac{\prod_{i=1}^{n}\left(\rho^{1-\mu_{i}^{q}}-1\right)^{\lambda_{i} \omega_{i}}}{(\rho-1)}\right)\right)^{\frac{1}{q}},\right. \\
\left.\left(\log _{\rho}\left(1+\frac{\prod_{i=1}^{n}\left(\rho^{v_{i}^{q}}-1\right)^{\lambda_{i} \omega_{i}}}{(\rho-1)}\right)\right)^{\frac{1}{q}}\right\rangle
\end{gathered}
$$


Remark 3 1. If $\rho \rightarrow 1$, then the Cq-ROFFWA operator reduces to the Cq-ROFWA operator [15];

2. If $\rho \rightarrow 1$ and $\lambda_{i}=1$, then the Cq-ROFFWA operator reduces to the q-ROFWA operator [21].

\subsection{ATS-Cq-ROFWG operator}

Definition 10 Let $\alpha_{i}=\left(\mu_{i}, v_{i}\right)(i=1,2, \ldots, n)$ be a collection of q-ROFNs. The ATSCq-ROFWG operator is denoted as

$$
A T S-C q-R O F W G\left(\left\langle\lambda_{1}, \alpha_{1}\right\rangle,\left\langle\lambda_{2}, \alpha_{2}\right\rangle, \ldots,\left\langle\lambda_{n}, \alpha_{n}\right\rangle\right)=\otimes_{i=1}^{n}\left(\alpha_{i}^{\lambda_{i}}\right)^{\omega_{i}}
$$

where $\lambda_{i}$ and $\omega_{i}$ be the confidence levels and weight vector of $\alpha_{i}$, respectively, and taking $0 \leq \lambda_{i} \leq 1, \sum_{i=1}^{n} \omega_{i}=1$ and $\omega_{i} \in(0,1)$.

Remark 4 If $\lambda_{1}=\lambda_{2}=\ldots=\lambda_{n}=1$, then the ATS-Cq-ROFWG operator reduces to the q-rung orthopair fuzzy weighted geometric operator based on ATS(ATS-qROFWG)

$$
A T S-q-R O F W G\left(\left\langle\alpha_{1}\right\rangle,\left\langle\alpha_{2}\right\rangle, \ldots,\left\langle\alpha_{n}\right\rangle\right)=\bigotimes_{i=1}^{n}\left(\alpha_{i}\right)^{\omega_{i}}
$$

Theorem 5 Let $\alpha_{i}=\left(\mu_{i}, v_{i}\right)(i=1,2, \ldots, n)$ be a collection of $q$-ROFNs, and $\lambda_{i}$ and $\omega_{i}$ be the confidence levels and weight vector of $\alpha_{i}$, respectively. then the aggregated value of $\alpha_{i}$ obtained by ATS-Cq-ROFWG operator is an q-ROFNs and

$$
\begin{aligned}
& \text { ATS-Cq-ROFWG }\left(\left\langle\lambda_{1}, \alpha_{1}\right\rangle,\left\langle\lambda_{2}, \alpha_{2}\right\rangle, \ldots,\left\langle\lambda_{n}, \alpha_{n}\right\rangle\right) \\
& =\otimes_{i=1}^{n}\left(\alpha_{i}^{\lambda_{i}}\right)^{\omega_{i}}=\left(g^{-1}\left(\sum_{i=1}^{n} \omega_{i} \lambda_{i} g\left(\mu_{i}\right)\right), h^{-1}\left(\sum_{i=1}^{n} \omega_{i} \lambda_{i} h\left(v_{i}\right)\right)\right)
\end{aligned}
$$

where $0 \leq \lambda_{i} \leq 1, \sum_{i=1}^{n} \omega_{i}=1$ and $\omega_{i} \in(0,1)$.

Proof. The Eq. (13) can be proved by mathematical induction.

(1) For $n=2$, we can get

$$
\begin{aligned}
& A T S-C q-R O F W G\left(\left\langle\lambda_{1}, \alpha_{1}\right\rangle,\left\langle\lambda_{2}, \alpha_{2}\right\rangle, \ldots,\left\langle\lambda_{n}, \alpha_{n}\right\rangle\right) \\
&=\stackrel{\otimes}{i=1}^{\otimes}\left(\alpha_{i}^{\lambda_{i}}\right)^{\omega_{i}}=\left(\alpha_{1}^{\lambda_{1}}\right)^{\omega_{1}} \otimes\left(\alpha_{2}^{\lambda_{2}}\right)^{\omega_{2}} \\
&=\left(g^{-1}\left(g\left(g^{-1}\left(\omega_{1} g\left(g^{-1}\left(\lambda_{1} g\left(\mu_{1}\right)\right)\right)\right)\right)+g\left(g^{-1}\left(\omega_{2} g\left(g^{-1}\left(\lambda_{2} g\left(\mu_{2}\right)\right)\right)\right)\right)\right),\right. \\
&\left.h^{-1}\left(h\left(h^{-1}\left(\omega_{1} h\left(h^{-1}\left(\lambda_{1} h\left(v_{1}\right)\right)\right)\right)\right)+h\left(h^{-1}\left(\omega_{2} h\left(h^{-1}\left(\lambda_{2} h\left(v_{2}\right)\right)\right)\right)\right)\right)\right) \\
&=\left(g^{-1}\left(\omega_{1} \lambda_{1} g\left(\mu_{1}\right)+\omega_{2} \lambda_{2} g\left(\mu_{2}\right)\right), h^{-1}\left(\omega_{1} \lambda_{1} h\left(v_{1}\right)+\omega_{2} \lambda_{2} h\left(v_{2}\right)\right)\right) .
\end{aligned}
$$

(2) Given Eq. (13) holds for $n=k$, we can get

$$
\text { ATS-Cq-ROFWG }\left(\left\langle\lambda_{1}, \alpha_{1}\right\rangle,\left\langle\lambda_{2}, \alpha_{2}\right\rangle, \ldots,\left\langle\lambda_{n}, \alpha_{n}\right\rangle\right)
$$


12

Y. Shao, N. Wang and Z. Gong

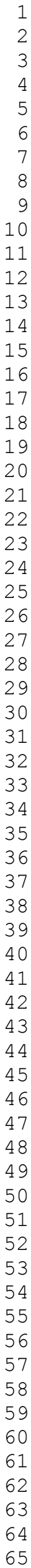

$$
=\otimes_{i=1}^{n}\left(\alpha_{i}^{\lambda_{i}}\right)^{\omega_{i}}=\left(g^{-1}\left(\sum_{i=1}^{k} \omega_{i} \lambda_{i} g\left(\mu_{i}\right)\right), h^{-1}\left(\sum_{i=1}^{k} \omega_{i} \lambda_{i} h\left(v_{i}\right)\right)\right) .
$$

(3) When $n=k+1$, we have

$$
\begin{aligned}
& A T S-C q-R O F W G\left(\left\langle\lambda_{1}, \alpha_{1}\right\rangle,\left\langle\lambda_{2}, \alpha_{2}\right\rangle, \ldots,\left\langle\lambda_{n}, \alpha_{n}\right\rangle\right) \\
&=\otimes_{i=1}^{\otimes}\left(\alpha_{i}^{\lambda_{i}}\right)^{\omega_{i}} \otimes\left(\alpha_{k+1} \lambda_{k+1}\right)^{\omega_{k+1}} \\
&=\left(g^{-1}\left(\sum_{i=1}^{k} \omega_{i} \lambda_{i} g\left(\mu_{i}\right)\right), h^{-1}\left(\sum_{i=1}^{k} \omega_{i} \lambda_{i} h\left(v_{i}\right)\right)\right) \otimes \\
&\left(g^{-1}\left(\omega_{k+1} \lambda_{k+1} g\left(\mu_{k+1}\right)\right), h^{-1}\left(\omega_{k+1} \lambda_{k+1} h\left(v_{k+1}\right)\right)\right) \\
&=\left(g^{-1}\left(g\left(g^{-1}\left(\sum_{i=1}^{k} \omega_{i} \lambda_{i} g\left(\mu_{i}\right)\right)\right)+g\left(g^{-1}\left(\omega_{k+1} \lambda_{k+1} g\left(\mu_{k+1}\right)\right)\right)\right),\right. \\
&\left.h^{-1}\left(h\left(h^{-1}\left(\sum_{i=1}^{k} \omega_{i} \lambda_{i} h\left(v_{i}\right)\right)\right)+h\left(h^{-1}\left(\omega_{k+1} \lambda_{k+1} h\left(v_{k+1}\right)\right)\right)\right)\right) \\
&=\left(g^{-1}\left(\sum_{i=1}^{k} \omega_{i} \lambda_{i} g\left(\mu_{i}\right)+\omega_{k+1} \lambda_{k+1} g\left(\mu_{k+1}\right)\right)\right. \\
&\left.h^{-1}\left(\sum_{i=1}^{k} \omega_{i} \lambda_{i} h\left(v_{i}\right)+\omega_{k+1} \lambda_{k+1} h\left(v_{k+1}\right)\right)\right) \\
&=\left(g^{-1}\left(\sum_{i=1}^{k+1} \omega_{i} \lambda_{i} g\left(\mu_{i}\right)\right), h^{-1}\left(\sum_{i=1}^{k+1} \omega_{i} \lambda_{i} h\left(v_{i}\right)\right)\right) .
\end{aligned}
$$

That is Eq. (13) holds for $n=k+1$.

Now, we prove ATS-Cq-ROFWG operator is an q-ROFNs.

As can be seen from above, AT function $g(t):[0,1] \rightarrow[0, \infty]$ is strictly decreasing, and AS function $h(t)$ is strictly increasing, simultaneously, $h(t)$ and $g(t)$ are satisfied $h(t)=g(1-t)$, hence

$$
0 \leq h^{-1}\left(\sum_{i=1}^{n} \omega_{i}\left(\lambda_{i} h\left(\mu_{i}\right)\right)\right), g^{-1}\left(\sum_{i=1}^{n} \omega_{i}\left(\lambda_{i} g\left(v_{i}\right)\right)\right) \leq 1
$$

and

$$
\begin{aligned}
& h^{-1}\left(\sum_{i=1}^{n} \omega_{i}\left(\lambda_{i} h\left(\mu_{i}\right)\right)\right)+g^{-1}\left(\sum_{i=1}^{n} \omega_{i}\left(\lambda_{i} g\left(v_{i}\right)\right)\right) \\
& \leq h^{-1}\left(\sum_{i=1}^{n} \omega_{i}\left(\lambda_{i} h\left(\mu_{i}\right)\right)\right)+g^{-1}\left(\sum_{i=1}^{n} \omega_{i}\left(\lambda_{i} g\left(1-\mu_{i}\right)\right)\right) \\
& =h^{-1}\left(\sum_{i=1}^{n} \omega_{i}\left(\lambda_{i} h\left(\mu_{i}\right)\right)\right)+1-h^{-1}\left(\sum_{i=1}^{n} \omega_{i}\left(\lambda_{i} h\left(\mu_{i}\right)\right)\right)
\end{aligned}
$$


$=1$.

Therefore, the proof is completed.

Next, similar to ATS-Cq-ROFWA, we present some basic properties of the ATSCq-ROFWG operator.

Theorem 6 (Idempotency) Let $\alpha_{i}=\left(\mu_{i}, v_{i}\right)$ be a collection of $q$-ROFNs and suppose $\left(\alpha, \alpha_{1}, \alpha_{2}, \ldots, \alpha_{n}\right)$ are equal, which is $\alpha=\alpha_{i}=(\mu, v)$, for $i=1,2, \ldots, n$, then

$$
A T S-C q-R O F W G\left(\left\langle\lambda_{1}, \alpha_{2}\right\rangle,\left\langle\lambda_{2}, \alpha_{2}\right\rangle, \ldots,\left\langle\lambda_{n}, \alpha_{n}\right\rangle\right)=\alpha^{\lambda}
$$

Theorem 7 (Monotonicity) Suppose $\alpha_{i}=\left(\alpha_{1}, \alpha_{2}, \ldots, \alpha_{n}\right), \ddot{\alpha}_{i}=\left(\ddot{\alpha}_{1}, \ddot{\alpha}_{2}, \ldots, \ddot{\alpha}_{n}\right)$ be two collections of $q$-ROFNs, when $\mu_{i} \leq \ddot{\mu}_{i}$ and $v_{i} \geq \ddot{v}_{i}$, we have

$$
\begin{aligned}
& A T S-C q-\operatorname{ROFWG}\left(\left\langle\lambda_{1}, \alpha_{1}\right\rangle,\left\langle\lambda_{2}, \alpha_{2}\right\rangle, \ldots,\left\langle\lambda_{n}, \alpha_{n}\right\rangle\right) \\
& \leq A T S-C q-\operatorname{ROFWG}\left(\left\langle\lambda_{1}, \ddot{\alpha}_{1}\right\rangle,\left\langle\lambda_{2}, \ddot{\alpha}_{2}\right\rangle, \ldots,\left\langle\lambda_{n}, \ddot{\alpha}_{n}\right\rangle\right) .
\end{aligned}
$$

Theorem 8 (Boundedness) Suppose $\ddot{\alpha}_{i}=\left(\ddot{\alpha}_{1}, \ddot{\alpha}_{2}, \ldots, \ddot{\alpha}_{n}\right)$ be a collection of $q$-ROFNs, $\ddot{\alpha}_{\min }=\left(\min _{i}\left\{\ddot{\mu}_{i}^{\lambda_{i}}\right\}, \max _{i}\left\{\ddot{v}_{i}^{\lambda_{i}}\right\}\right)$, and $\ddot{\alpha}_{\max }=\left(\max _{i}\left\{\ddot{\mu}_{i}^{\lambda_{i}}\right\}, \min _{i}\left\{\ddot{v}_{i}^{\lambda_{i}}\right\}\right)$, then we have

$$
\ddot{\alpha}_{\min } \leq A T S-C q-R O F W G\left(\left\langle\lambda_{1}, \ddot{\alpha}_{1}\right\rangle,\left\langle\lambda_{2}, \ddot{\alpha}_{2}\right\rangle, \ldots,\left\langle\lambda_{n}, \ddot{\alpha}_{n}\right\rangle\right) \leq \ddot{\alpha}_{\max }
$$

Next, we give some series of special AOs for different additive generator $g(t)$.

(1) If $g(t)=-\log \left(t^{q}\right)$, we can get confidence q-rung orthopair fuzzy algebraic weighted geometric $(\mathrm{Cq}-\mathrm{ROFWG})[15]$ operator, that is

$$
\begin{gathered}
C q-\operatorname{ROFWG}\left(\left\langle\lambda_{1}, \alpha_{1}\right\rangle,\left\langle\lambda_{2}, \alpha_{2}\right\rangle, \ldots,\left\langle\lambda_{n}, \alpha_{n}\right\rangle\right) \\
=\left\langle\prod_{i=1}^{n}\left(\mu_{i}\right)^{\lambda_{i} \omega_{i}},\left(1-\prod_{i=1}^{n}\left(1-v_{i}^{q}\right)^{\lambda_{i} \omega_{i}}\right)^{\frac{1}{q}}\right\rangle
\end{gathered}
$$

(2) If $g(t)=\log \left(\frac{2-t^{q}}{t^{q}}\right)$, we can get confidence q-rung orthopair fuzzy Einstein weighted geometric (Cq-ROFEWG) operator, that is

$$
\begin{aligned}
& C q-\operatorname{ROFEWG}\left(\left\langle\lambda_{1}, \alpha_{1}\right\rangle,\left\langle\lambda_{2}, \alpha_{2}\right\rangle, \ldots,\left\langle\lambda_{n}, \alpha_{n}\right\rangle\right) \\
& =\left\langle\left(\frac{2 \prod_{i=1}^{n}\left(\mu_{i}^{q}\right)^{\lambda_{i} \omega_{i}}}{\prod_{i=1}^{n}\left(2-\mu_{i}^{q}\right)^{\lambda_{i} \omega_{i}}+\prod_{i=1}^{n}\left(\mu_{i}^{q}\right)^{\lambda_{i} \omega_{i}}}\right)^{\frac{1}{q}},\left(\frac{\prod_{i=1}^{n}\left(1+v_{i}^{q}\right)^{\lambda_{i} \omega_{i}}+\left(1+v_{i}^{q}\right)^{\lambda_{i} \omega_{i}}}{\prod_{i=1}^{n}\left(1+v_{i}^{q}\right)^{\lambda_{i} \omega_{i}}+\left(1+v_{i}^{q}\right)^{\lambda_{i} \omega_{i}}}\right)^{\frac{1}{q}}\right\rangle .
\end{aligned}
$$


(3) If $g(t)=\log \left(\frac{\rho+(1-\rho) t^{q}}{t^{q}}\right)$, we can get confidence q-rung orthopair fuzzy Hammer weighted geometric (Cq-ROFHWG) operator, that is

$$
\begin{aligned}
& \operatorname{Cq}-\operatorname{ROFHWG}\left(\left\langle\lambda_{1}, \alpha_{1}\right\rangle,\left\langle\lambda_{2}, \alpha_{2}\right\rangle, \ldots,\left\langle\lambda_{n}, \alpha_{n}\right\rangle\right) \\
= & \left\langle\left(\frac{\rho \prod_{i=1}^{n}(a)^{\lambda_{i} \omega_{i}}}{\prod_{i=1}^{n}(b)^{\lambda_{i} \omega_{i}}+(\rho-1) \prod_{i=1}^{n}(a)^{\lambda_{i} \omega_{i}}}\right)^{\frac{1}{q}},\left(\frac{\prod_{i=1}^{n}(c)^{\lambda_{i} \omega_{i}}-\prod_{i=1}^{n}(d)^{\lambda_{i} \omega_{i}}}{\prod_{i=1}^{n}(c)^{\lambda_{i} \omega_{i}}+(\rho-1) \prod_{i=1}^{n}(d)^{\lambda_{i} \omega_{i}}}\right)^{\frac{1}{q}}\right\rangle .
\end{aligned}
$$

where $a=\mu_{i}^{q}, b=1+(\rho-1)\left(1-\mu_{i}^{q}\right), c=1+(\rho-1) v_{i}^{q}, d=1-v_{i}^{q}$.

Remark 5 1. If $\rho=1$, then the Cq-ROFHWG operator reduces to the Cq-ROFWG operator [15];

2. If $\rho=2$, then the Cq-ROFHWG operator reduces to the Cq-ROFEWG operator;

3. If $\rho=1$ and $\lambda_{i}=1$, then the Cq-ROFHWG operator reduces to the q-ROFWG operator [21];

4. If $q=2, \rho=2$ and $\lambda_{i}=1$, then the Cq-ROFHWG operator reduces to the PFEWG operator [10].

(4) If $g(t)=\log \left(\frac{\rho-1}{\rho^{t^{q}}-1}\right)$, we can get confidence q-rung orthopair fuzzy Frank weighted geometric (Cq-ROFFWG) operator, that is

$$
\begin{aligned}
& C q-\operatorname{ROFFWG}\left(\left\langle\lambda_{1}, \alpha_{1}\right\rangle,\left\langle\lambda_{2}, \alpha_{2}\right\rangle, \ldots,\left\langle\lambda_{n}, \alpha_{n}\right\rangle\right) \\
& =\left\langle\left(\log _{\rho}\left(1+\frac{\prod_{i=1}^{n}\left(\rho^{\mu_{i}^{q}}-1\right)^{\lambda_{i} \omega_{i}}}{(\rho-1)}\right)\right)^{\frac{1}{q}}\right. \text {, } \\
& \left.\left(1-\log _{\rho}\left(1+\frac{\prod_{i=1}^{n}\left(\rho^{1-v_{i}^{q}}-1\right)^{\lambda_{i} \omega_{i}}}{(\rho-1)}\right)\right)^{\frac{1}{q}}\right\rangle
\end{aligned}
$$

Remark 6 1. If $\rho \rightarrow 1$, then the Cq-ROFFWG operator reduces to the $\mathrm{Cq}-\mathrm{ROFWG}$ operator [15];

2. If $\rho \rightarrow 1$ and $\lambda_{i}=1$, then the Cq-ROFFWG operator reduces to the q-ROFWG operator [21].

\subsection{ATS-Cq-ROFOWA operator and ATS-Cq-ROFOWG operator}

Definition 11 Let $\alpha_{i}=\left(\mu_{i}, v_{i}\right)(i=1,2, \ldots, n)$ be a collection of q-ROFNs. The ATSCq-ROFOWA operator is denoted as

$$
A T S-C q-\operatorname{ROFOWA}\left(\left\langle\lambda_{1}, \alpha_{1}\right\rangle,\left\langle\lambda_{2}, \alpha_{2}\right\rangle, \ldots,\left\langle\lambda_{n}, \alpha_{n}\right\rangle\right)=\bigoplus_{i=1}^{n} \omega_{i}\left(\lambda_{\sigma_{(i)}} \alpha_{\sigma_{(i)}}\right)
$$


and the ATS-Cq-ROFOWG operator is denoted as

$$
\text { ATS-Cq-ROFOWG }\left(\left\langle\lambda_{1}, \alpha_{1}\right\rangle,\left\langle\lambda_{2}, \alpha_{2}\right\rangle, \ldots,\left\langle\lambda_{n}, \alpha_{n}\right\rangle\right)=\bigotimes_{i=1}^{n}\left(\alpha_{\sigma_{(i)}} \lambda_{\sigma_{(i)}}\right)^{\omega_{i}}
$$

where $\lambda_{i}$ and $\omega_{i}$ be the confidence levels and associated weight vector of $\alpha_{i}$, respectively, and taking $0 \leq \lambda_{i} \leq 1, \sum_{i=1}^{n} \omega_{i}=1$ and $\omega_{i} \in(0,1)$. In addition, $\lambda_{\sigma_{(i)}} \alpha_{\sigma_{(i)}}$ and $\alpha_{\sigma_{(i)}}{ }^{\lambda_{\sigma_{(i)}}}$ be the $i$ th largest of $\lambda_{i} \alpha_{i}$ and $\alpha_{i}^{\lambda_{i}}$, respectively.

Theorem 9 Let $\alpha_{i}=\left(\mu_{i}, v_{i}\right)(i=1,2, \ldots, n)$ be a collection of $q$-ROFNs, and $\lambda_{i}$ and $\omega_{i}$ be the confidence levels and weight vector of $\alpha_{i}$, respectively, where $0 \leq \lambda_{i} \leq 1$, $\sum_{i=1}^{n} \omega_{i}=1$ and $\omega_{i} \in(0,1)$. In addition, $\lambda_{\sigma_{(i)}} \alpha_{\sigma_{(i)}}$ and $\alpha_{\sigma_{(i)}} \lambda_{\sigma_{(i)}}$ be the ith largest of $\lambda_{i} \alpha_{i}$ and $\alpha_{i}^{\lambda_{i}}$, respectively, then the aggregated value of $\alpha_{i}$ obtained by ATS-Cq-ROFOWA operator is an q-ROFNs and

$$
\begin{aligned}
& \text { ATS-Cq-ROFOWA }\left(\left\langle\lambda_{1}, \alpha_{1}\right\rangle,\left\langle\lambda_{2}, \alpha_{2}\right\rangle, \ldots,\left\langle\lambda_{n}, \alpha_{n}\right\rangle\right)=\stackrel{\oplus}{i=1}_{\oplus}^{n} \omega_{i}\left(\lambda_{\sigma_{(i)}} \alpha_{\sigma_{(i)}}\right) \\
& =\left(h^{-1}\left(\sum_{i=1}^{n} \omega_{i}\left(\lambda_{\sigma_{(i)}} h\left(\mu_{\sigma_{(i)}}\right)\right)\right), g^{-1}\left(\sum_{i=1}^{n} \omega_{i}\left(\lambda_{\sigma_{(i)}} g\left(v_{\sigma_{(i)}}\right)\right)\right)\right) .
\end{aligned}
$$

In addition, the aggregated value of $\alpha_{i}$ obtained by ATS-Cq-ROFOWG operator is also an $q$-ROFNs and

$$
\begin{aligned}
& \text { ATS-Cq-ROFOWG }\left(\left\langle\lambda_{1}, \alpha_{1}\right\rangle,\left\langle\lambda_{2}, \alpha_{2}\right\rangle, \ldots,\left\langle\lambda_{n}, \alpha_{n}\right\rangle\right)={\stackrel{i=1}{\otimes}\left(\alpha_{\sigma_{(i)}} \lambda_{\sigma_{(i)}}\right)^{\omega_{i}}}\left(g^{-1}\left(\sum_{i=1}^{n} \omega_{i}\left(\lambda_{\sigma_{(i)}} g\left(\mu_{\sigma_{(i)}}\right)\right)\right), h^{-1}\left(\sum_{i=1}^{n} \omega_{i}\left(\lambda_{\sigma_{(i)}} h\left(v_{\sigma_{(i)}}\right)\right)\right)\right) .
\end{aligned}
$$

Proof. Similar to Theorem 1 and Theorem 5, so we omit it.

Similarly, we can get the same properties as ATS-Cq-ROFWA or ATS-Cq-ROFWG, so we omit it. In addition, we also give some series of special AOs for different additive generator $g(t)$.

(1) If $g(t)=-\log \left(t^{q}\right)$, we can get confidence q-rung orthopair fuzzy algebraic ordered weighted averaging (Cq-ROFOWA) operator [15], that is

$$
\begin{aligned}
& \text { Cq-ROFOWA }\left(\left\langle\lambda_{1}, \alpha_{1}\right\rangle,\left\langle\lambda_{2}, \alpha_{2}\right\rangle, \ldots,\left\langle\lambda_{n}, \alpha_{n}\right\rangle\right) \\
& =\left\langle\left(1-\prod_{i=1}^{n}\left(1-\mu_{\sigma(i)}^{q}\right)^{\lambda_{i} \omega_{i}}\right)^{\frac{1}{q}}, \prod_{i=1}^{n}\left(v_{\sigma(i)}\right)^{\lambda_{i} \omega_{i}}\right\rangle .
\end{aligned}
$$

And we can get confidence q-rung orthopair fuzzy algebraic ordered weighted geometric (Cq-ROFOWG) operator [15], that is

$$
\begin{aligned}
& \text { Cq-ROFOWG }\left(\left\langle\lambda_{1}, \alpha_{1}\right\rangle,\left\langle\lambda_{2}, \alpha_{2}\right\rangle, \ldots,\left\langle\lambda_{n}, \alpha_{n}\right\rangle\right) \\
& =\left\langle\prod_{i=1}^{n}\left(\mu_{\sigma(i)}\right)^{\lambda_{\sigma(i)} \omega_{i}},\left(1-\prod_{i=1}^{n}\left(1-v_{\sigma(i)}^{q}\right)^{\lambda_{\sigma(i)} \omega_{i}}\right)^{\frac{1}{q}}\right\rangle .
\end{aligned}
$$


(2) If $g(t)=\log \left(\frac{2-t^{q}}{t^{q}}\right)$, we can get confidence q-rung orthopair fuzzy Einstein ordered weighted averaging $(\mathrm{Cq}-\mathrm{ROFEOWA})$ operator, that is

$$
\begin{gathered}
\text { Cq-ROFEOWA }\left(\left\langle\lambda_{1}, \alpha_{1}\right\rangle,\left\langle\lambda_{2}, \alpha_{2}\right\rangle, \ldots,\left\langle\lambda_{n}, \alpha_{n}\right\rangle\right) \\
=\left\langle\left(\frac{\prod_{i=1}^{n}\left(1+\mu_{\sigma(i)}^{q}\right)^{\lambda_{\sigma(i)} \omega_{i}}+\left(1+\mu_{\sigma(i)}^{q}\right)^{\lambda_{\sigma(i)} \omega_{i}}}{\prod_{i=1}^{n}\left(1+\mu_{\sigma(i)}^{q}\right)^{\lambda_{\sigma(i)} \omega_{i}}+\left(1+\mu_{\sigma(i)}^{q}\right)^{\lambda_{\sigma(i)} \omega_{i}}}\right)^{\frac{1}{q}},\right. \\
\left.\left(\frac{2 \prod_{i=1}^{n}\left(v_{\sigma(i)}\right)^{\lambda_{\sigma(i)} \omega_{i}}}{\prod_{i=1}^{n}\left(2-v_{\sigma(i)}\right)^{\lambda_{\sigma(i)} \omega_{i}}+\prod_{i=1}^{n}\left(v_{\sigma(i)}\right)^{\lambda_{\sigma(i)} \omega_{i}}}\right)^{\frac{1}{q}}\right\rangle .
\end{gathered}
$$

And we can get confidence q-rung orthopair fuzzy Einstein ordered weighted geometric (Cq-ROFEOWG) operator, that is

$$
\begin{aligned}
& \text { Cq-ROFEOWG }\left(\left\langle\lambda_{1}, \alpha_{1}\right\rangle,\left\langle\lambda_{2}, \alpha_{2}\right\rangle, \ldots,\left\langle\lambda_{n}, \alpha_{n}\right\rangle\right) \\
& =\left\langle\left(\frac{2 \prod_{i=1}^{n}\left(\mu_{\sigma(i)}^{q}\right)^{\lambda_{\sigma(i)} \omega_{i}}}{\prod_{i=1}^{n}\left(2-\mu_{\sigma(i)}^{q}\right)^{\lambda_{\sigma(i)} \omega_{i}}+\prod_{i=1}^{n}\left(\mu_{\sigma(i)}^{q}\right)^{\lambda_{\sigma(i)} \omega_{i}}}\right)^{\frac{1}{q}},\right. \\
& \\
& \left.\left(\frac{\prod_{i=1}^{n}\left(1+v_{\sigma(i)}^{q}\right)^{\lambda_{\sigma(i)} \omega_{i}}+\left(1+v_{\sigma(i)}^{q}\right)^{\lambda_{\sigma(i)} \omega_{i}}}{\prod_{i=1}^{n}\left(1+v_{\sigma(i)}^{q}\right)^{\lambda_{\sigma(i)} \omega_{i}}+\left(1+v_{\sigma(i)}^{q}\right)^{\lambda_{\sigma(i)} \omega_{i}}}\right)\right\rangle .
\end{aligned}
$$

(3) If $g(t)=\log \left(\frac{\rho+(1-\rho) t^{q}}{t^{q}}\right)$, we can get confidence q-rung orthopair fuzzy Hammer ordered weighted averaging (Cq-ROFHOWA) operator, that is

$$
\begin{gathered}
\text { Cq-ROFHOWA }\left(\left\langle\lambda_{1}, \alpha_{1}\right\rangle,\left\langle\lambda_{2}, \alpha_{2}\right\rangle, \ldots,\left\langle\lambda_{n}, \alpha_{n}\right\rangle\right) \\
=\left\langle\left(\frac{\prod_{i=1}^{n}(a)^{\lambda_{\sigma(i)} \omega_{i}}-\prod_{i=1}^{n}(b)^{\lambda_{\sigma(i)} \omega_{i}}}{\prod_{i=1}^{n}(a)^{\lambda_{\sigma(i)} \omega_{i}}+(\rho-1) \prod_{i=1}^{n}(b)^{\lambda_{\sigma(i)} \omega_{i}}}\right)^{\frac{1}{q}}\right. \\
\left.\left(\frac{\rho \prod_{i=1}^{n}(c)^{\lambda_{\sigma(i)} \omega_{i}}}{\prod_{i=1}^{n}(d)^{\lambda_{i} \omega_{i}}+(\rho-1) \prod_{i=1}^{n}(c)^{\lambda_{\sigma(i)} \omega_{i}}}\right)^{\frac{1}{q}}\right\rangle
\end{gathered}
$$

where $a_{1}=1+(\rho-1) \mu_{\sigma(i)}^{q}, b_{1}=1-\mu_{\sigma(i)}^{q}, c_{1}=v_{\sigma(i)}^{q}, d_{1}=1+(\rho-1)\left(1-v_{\sigma(i)}^{q}\right)$.

Remark 7 1. If $\rho=1$, then the Cq-ROFHOWA operator reduces to the Cq-ROFOWA operator [15]; 
2. If $\rho=2$, then the Cq-ROFHOWA operator reduces to the Cq-ROFEOWA operator;

3. If $q=2, \rho=1$ and $\lambda_{i}=1$, then the Cq-ROFHOWA operator reduces to the PFOWA operator [9];

4. If $q=2, \rho=2$ and $\lambda_{i}=1$, then the Cq-ROFHOWA operator reduces to the PFEOWA operator [9].

In addition, we also can get confidence q-rung orthopair fuzzy Hammer ordered weighted geometric (Cq-ROFHOWG) operator, that is

$$
\begin{aligned}
& \text { Cq-ROFHOWG }\left(\left\langle\lambda_{1}, \alpha_{1}\right\rangle,\left\langle\lambda_{2}, \alpha_{2}\right\rangle, \ldots,\left\langle\lambda_{n}, \alpha_{n}\right\rangle\right) \\
& =\left\langle\left(\frac{\rho \prod_{i=1}^{n}(a)^{\lambda_{\sigma(i)} \omega_{i}}}{\prod_{i=1}^{n}(b)^{\lambda_{i} \omega_{i}}+(\rho-1) \prod_{i=1}^{n}(a)^{\lambda_{\sigma(i)} \omega_{i}}}\right)^{\frac{1}{q}}\right. \\
& \\
& \left.\left(\frac{\prod_{i=1}^{n}(c)^{\lambda_{\sigma(i)} \omega_{i}}-\prod_{i=1}^{n}(d)^{\lambda_{\sigma(i)} \omega_{i}}}{\prod_{i=1}^{n}(c)^{\lambda_{\sigma(i)} \omega_{i}}+(\rho-1) \prod_{i=1}^{n}(d)^{\lambda_{\sigma(i)} \omega_{i}}}\right)^{\frac{1}{q}}\right\rangle .
\end{aligned}
$$

where $a_{2}=\mu_{\sigma(i)}^{q}, b_{2}=1+(\rho-1)\left(1-\mu_{\sigma(i)}^{q}\right), c_{2}=1+(\rho-1) v_{\sigma(i)}^{q}, d_{2}=1-v_{\sigma(i)}^{q}$.

Remark 8 1. If $\rho=1$, then the Cq-ROFHOWG operator reduces to the Cq-ROFOWG operator [15];

2. If $\rho=2$, then the Cq-ROFHOWG operator reduces to the Cq-ROFEOWG operator;

3. If $q=2, \rho=1$ and $\lambda_{i}=1$, then the Cq-ROFHOWG operator reduces to the PFOWG operator [10];

4. If $q=2, \rho=2$ and $\lambda_{i}=1$, then the Cq-ROFHOWG operator reduces to the PFEOWG operator [10].

(4) If $g(t)=\log \left(\frac{\rho-1}{\rho^{t q}-1}\right)$, we can get confidence q-rung orthopair fuzzy Frank ordered weighted averaging (Cq-ROFFOWA) operator, that is

$$
\begin{aligned}
& \text { Cq-ROFFOWA }\left(\left\langle\lambda_{1}, \alpha_{1}\right\rangle,\left\langle\lambda_{2}, \alpha_{2}\right\rangle, \ldots,\left\langle\lambda_{n}, \alpha_{n}\right\rangle\right) \\
& =\left\langle\left(1-\log _{\rho}\left(1+\frac{\prod_{i=1}^{n}\left(\rho^{1-\mu_{\sigma(i)}^{q}}-1\right)^{\lambda_{\sigma(i)} \omega_{i}}}{(\rho-1)}\right)\right)^{\frac{1}{q}},\right. \\
& \left.\left(\log _{\rho}\left(1+\frac{\prod_{i=1}^{n}\left(\rho^{v_{\sigma(i)}^{q}}-1\right)^{\lambda_{\sigma(i)} \omega_{i}}}{(\rho-1)}\right)\right)^{\frac{1}{q}}\right\rangle .
\end{aligned}
$$

Remark 9 1. If $\rho \rightarrow 1$, then the Cq-ROFFOWA operator reduces to the Cq-ROFOWA operator [15]; 
2. If $q=2, \rho \rightarrow 1$ and $\lambda_{i}=1$, then the Cq-ROFFOWA operator reduces to the PFOWA operator [9].

In addition, we can get confidence q-rung orthopair fuzzy Frank ordered weighted geometric (Cq-ROFFOWG) operator, that is

$$
\begin{aligned}
& \text { Cq-ROFFOW }\left(\left\langle\lambda_{1}, \alpha_{1}\right\rangle,\left\langle\lambda_{2}, \alpha_{2}\right\rangle, \ldots,\left\langle\lambda_{n}, \alpha_{n}\right\rangle\right) \\
& =\left\langle\left(\log _{\rho}\left(1+\frac{\prod_{i=1}^{n}\left(\rho^{\mu_{\sigma(i)}^{q}}-1\right)^{\lambda_{\sigma(i)} \omega_{i}}}{(\rho-1)}\right)\right)^{\frac{1}{q}},\right. \\
& \left.\left(1-\log _{\rho}\left(1+\frac{\prod_{i=1}^{n}\left(\rho^{1-v_{\sigma(i)}^{q}}-1\right)^{\lambda_{\sigma(i)} \omega_{i}}}{(\rho-1)}\right)\right)^{\frac{1}{q}}\right\rangle .
\end{aligned}
$$

Remark 10 1. If $\rho \rightarrow 1$, then the Cq-ROFFOWG operator reduces to the Cq-ROFOWG operator [15];

2. If $q=2, \rho \rightarrow 1$ and $\lambda_{i}=1$, then the Cq-ROFFOWG operator reduces to the PFOWG operator [10].

\section{MCGDM approach based on ATS-Cq-ROF information aggregation operators}

MCGDM is widely applied in many areas of real life, and the correct and reasonable decision-making result is pursued by the decision maker. Therefore, decision-making method is particularly important, and different decision-making methods should be selected in different decision situations. For this, in this section, we propose an approach to solve the MCGDM problem based on the proposed AOs theory.

Let $e=\left\{e_{1}, e_{2}, \ldots, e_{c}\right\}$ be a set of experts, whose weight vector is $\omega_{k}(k=1,2, \ldots, t)$. Let $A_{i}(i=1,2, \ldots, m)$ are $m$ alternatives, and $G_{j}(j=1,2, \ldots, n)$ are $n$ criteria whose weight vector is $w_{j}(j=1,2, \ldots, n)$, satisfying $w_{j}>0, \sum_{j=1}^{n} w_{j}=1$. Each expert gives the decision matrix $Q^{p}=\left\langle\lambda_{i j}^{p},\left(\mu_{i j}^{p}, v_{i j}^{p}\right)\right\rangle_{m \times n}$ for $i=1,2, \ldots, m ; j=1,2, \ldots, n$ and $p=1,2, \ldots, l$, which contains the confidence levels values that take into account their familiarity with the evaluation fields and the evaluation values of $m$ alternatives under $n$ criteria simultaneously. In the following, specific decision steps are given.

Step 1. From the outset, acquire the normalized q-rung orthopair fuzzy decision matrix. As is known to all, the attributes set is generally presented in two opposite sorts. On the one hand, the beneficial type that has a positive effect on the outcomes. On the other hand, the cost type that has a negative effect on the outcomes. If there are cost criteria values, then we need to convert them to benefit criteria values by the following formula. Otherwise, this step can be neglected.

$$
\tilde{\xi}_{i j}^{t}=\left\{\begin{array}{l}
\left(\mu_{i j}^{t}, v_{i j}^{t}\right), \text { for benefit criteria } G_{j} \\
\left(\mu_{i j}^{t}, v_{i j}^{t}\right)^{c}, \text { for cost criteria } G_{j} .
\end{array}\right.
$$


Step 2. Compose all the q-rung orthopair fuzzy decision matrices to the integrated decision matrix $Q$ by using the four kinds of the proposed AOs:

$$
A T S-C q-R O F W A\left(\left\langle\lambda_{1}, \alpha_{1}\right\rangle,\left\langle\lambda_{2}, \alpha_{2}\right\rangle, \ldots,\left\langle\lambda_{n}, \alpha_{n}\right\rangle\right)=\bigoplus_{i=1}^{n} \omega_{i}\left(\lambda_{i} \alpha_{i}\right)
$$

Or

$$
A T S-C q-R O F W G\left(\left\langle\lambda_{1}, \alpha_{1}\right\rangle,\left\langle\lambda_{2}, \alpha_{2}\right\rangle, \ldots,\left\langle\lambda_{n}, \alpha_{n}\right\rangle\right)=\otimes_{i=1}^{n}\left(\alpha_{i}^{\lambda_{i}}\right)^{\omega_{i}}
$$

or

$$
A T S-C q-\operatorname{ROFOWA}\left(\left\langle\lambda_{1}, \alpha_{1}\right\rangle,\left\langle\lambda_{2}, \alpha_{2}\right\rangle, \ldots,\left\langle\lambda_{n}, \alpha_{n}\right\rangle\right)=\bigoplus_{i=1}^{n} \omega_{i}\left(\lambda_{\sigma_{(i)}} \alpha_{\sigma_{(i)}}\right)
$$

or

$$
A T S-C q-\operatorname{ROFOWG}\left(\left\langle\lambda_{1}, \alpha_{1}\right\rangle,\left\langle\lambda_{2}, \alpha_{2}\right\rangle, \ldots,\left\langle\lambda_{n}, \alpha_{n}\right\rangle\right)=\bigotimes_{i=1}^{n}\left(\alpha_{\sigma_{(i)}}^{\lambda_{\sigma_{(i)}}}\right)^{\omega_{i}} .
$$

Among them, the ordered weighted AOs is the evaluation value of the priority aggregation with high the confidence levels.

Step 3. Based on the collective decision matrix $Q$ of Step 2, the evaluation values $\phi_{i}(i=1, \ldots, m)$ of each alternative under various criteria are aggregated by using two kinds of information AOs:

$$
A T S-q-R O F W A\left(\alpha_{1}, \alpha_{2}, \ldots, \alpha_{n}\right)=\bigoplus_{i=1}^{n} \omega_{i}\left(\alpha_{i}\right)
$$

or

$$
A T S-q-R O F W G\left(\alpha_{1}, \alpha_{2}, \ldots, \alpha_{n}\right)=\otimes_{i=1}^{n}\left(\alpha_{i}\right)^{\omega_{i}} .
$$

Step 4. Based on the evaluation values $\phi_{i}$ obtained in Step 3, we can take advantage of the score function Eq. (2) and accuracy function Eq. (3) to compute the score values and accuracy values $S\left(\phi_{i}\right)(i=1, \ldots, m)$ of each alternative.

Step 5. According to the score value or accuracy value, rank the alternatives and compare them to select the best alternative.

\section{Application example on MCGDMs}

\subsection{Application of the defined MCGDM approach}

India's shipbuilding industry plans to buy a batch of welding robots for shipbuilding, ship repair, offshore engineering repair, and electromechanical equipment repair. At present, there are five robot companies $A=\left\{A_{1}, A_{2}, A_{3}, A_{4}, A_{5}\right\}$ to be selected, and their criteria have been investigated from six aspects, namely, load capacity $\left(G_{1}\right)$, welding quality $\left(G_{2}\right)$, life $\left(G_{4}\right)$, brand $\left(G_{4}\right)$, welding efficiency $\left(G_{5}\right)$, after-sale service $\left(G_{6}\right)$. Suppose that $\omega=(0.36,0.35,0.29)$ is the weight vector of a set of experts $e=$ $\left\{e_{1}, e_{2}, e_{3}\right\}$, attribute weight is $w=(0.15,0.24,0.14,0.17,0.19,0.11)$. Three experts gave the evaluation value for each attribute value of the three alternatives, and gave 
Table 1 q-rung orthopair fuzzy decision matrix $Q_{1}$

\begin{tabular}{ccccccc}
\hline & $G_{1}$ & $G_{2}$ & $G_{3}$ & $G_{4}$ & $G_{5}$ & $G_{6}$ \\
\hline$A_{1}$ & $\langle 0.70,(0.41,0.48)\rangle$ & $\langle 0.74,(0.52,0.30)\rangle$ & $\langle 0.70,(0.31,0.72)\rangle$ & $\langle 0.80,(0.82,0.12)\rangle$ & $\langle 0.70,(0.63,0.21)\rangle$ & $\langle 0.70,(0.51,0.30)\rangle$ \\
\hline$A_{2}$ & $\langle 0.75,(0.61,0.31)\rangle$ & $\langle 0.81,(0.50,0.20)\rangle$ & $\langle 0.74,(0.60,0.12)\rangle$ & $\langle 0.81,(0.71,0.12)\rangle$ & $\langle 0.72,(0.31,0.60)\rangle$ & $\langle 0.71,(0.39,0.30)\rangle$ \\
\hline$A_{3}$ & $\langle 0.74,(0.42,0.41)\rangle$ & $\langle 0.73,(0.78,0.11)\rangle$ & $\langle 0.79,(0.52,0.10)\rangle$ & $\langle 0.71,(0.60,0.31)\rangle$ & $\langle 0.82,(0.41,0.51)\rangle$ & $\langle 0.72,(0.30,0.23)\rangle$ \\
\hline$A_{4}$ & $\langle 0.71,(0.23,0.41)\rangle$ & $\langle 0.78,(0.41,0.30)\rangle$ & $\langle 0.75,(0.89,0.11)\rangle$ & $\langle 0.70,(0.71,0.22)\rangle$ & $\langle 0.74,(0.20,0.51)\rangle$ & $\langle 0.74,(0.70,0.10)\rangle$ \\
\hline$A_{5}$ & $\langle 0.81,(0.60,0.21)\rangle$ & $\langle 0.86,(0.30,0.60)\rangle$ & $\langle 0.80,(0.40,0.52)\rangle$ & $\langle 0.74,(0.30,0.50)\rangle$ & $\langle 0.70,(0.60,0.40)\rangle$ & $\langle 0.75,(0.90,0.12)\rangle$ \\
\hline
\end{tabular}

Table 2 q-rung orthopair fuzzy decision matrix $Q_{2}$

\begin{tabular}{ccccccc}
\hline & $G_{1}$ & $G_{2}$ & $G_{3}$ & $G_{4}$ & $G_{5}$ & $G_{6}$ \\
\hline$A_{1}$ & $\langle 0.81,(0.52,0.41)\rangle$ & $\langle 0.85,(0.29,0.42)\rangle$ & $\langle 0.71,(0.71,0.21)\rangle$ & $\langle 0.75,(0.89,0.11)\rangle$ & $\langle 0.82,(0.48,0.52)\rangle$ & $\langle 0.80,(0.31,0.60)\rangle$ \\
\hline$A_{2}$ & $\langle 0.70,(0.20,0.42)\rangle$ & $\langle 0.88,(0.51,0.23)\rangle$ & $\langle 0.85,(0.78,0.12)\rangle$ & $\langle 0.84,(0.70,0.11)\rangle$ & $\langle 0.89,(0.50,0.52)\rangle$ & $\langle 0.81,(0.68,0.20)\rangle$ \\
\hline$A_{3}$ & $\langle 0.75,(0.79,0.21)\rangle$ & $\langle 0.84,(0.60,0.31)\rangle$ & $\langle 0.85,(0.71,0.10)\rangle$ & $\langle 0.89,(0.70,0.11)\rangle$ & $\langle 0.85,(0.90,0.11)\rangle$ & $\langle 0.83,(0.71,0.20)\rangle$ \\
\hline$A_{4}$ & $\langle 0.85,(0.80,0.12)\rangle$ & $\langle 0.71,(0.42,0.52)\rangle$ & $\langle 0.70,(0.80,0.21)\rangle$ & $\langle 0.82,(0.42,0.60)\rangle$ & $\langle 0.81,(0.50,0.50)\rangle$ & $\langle 0.75,(0.51,0.41)\rangle$ \\
\hline$A_{5}$ & $\langle 0.91,(0.68,0.22)\rangle$ & $\langle 0.85,(0.60,0.39)\rangle$ & $\langle 0.87,(0.88,0.10)\rangle$ & $\langle 0.85,(0.60,0.40)\rangle$ & $\langle 0.90,(0.81,0.22)\rangle$ & $\langle 0.72,(0.42,0.52)\rangle$ \\
\hline
\end{tabular}

Table 3 q-rung orthopair fuzzy decision matrix $Q_{3}$

\begin{tabular}{ccccccc}
\hline & $G_{1}$ & $G_{2}$ & $G_{3}$ & $G_{4}$ & $G_{5}$ & $G_{6}$ \\
\hline$A_{1}$ & $\langle 0.73,(0.61,0.12)\rangle$ & $\langle 0.75,(0.80,0.11)\rangle$ & $\langle 0.80,(0.91,0.10)\rangle$ & $\langle 0.76,(0.60,0.30)\rangle$ & $\langle 0.81,(0.42,0.50)\rangle$ & $\langle 0.75,(0.90,0.13)\rangle$ \\
\hline$A_{2}$ & $\langle 0.81,(0.72,0.20)\rangle$ & $\langle 0.75,(0.51,0.50)\rangle$ & $\langle 0.78,(0.90,0.11)\rangle$ & $\langle 0.85,(0.41,0.50)\rangle$ & $\langle 0.75,(0.38,0.30)\rangle$ & $\langle 0.80,(0.80,0.11)\rangle$ \\
\hline$A_{3}$ & $\langle 0.84,(0.82,0.10)\rangle$ & $\langle 0.85,(0.90,0.10)\rangle$ & $\langle 0.90,(0.61,0.31)\rangle$ & $\langle 0.73,(0.52,0.50)\rangle$ & $\langle 0.80,(0.80,0.10)\rangle$ & $\langle 0.70,(0.43,0.61)\rangle$ \\
\hline$A_{4}$ & $\langle 0.75,(0.30,0.50)\rangle$ & $\langle 0.85,(0.62,0.31)\rangle$ & $\langle 0.90,(0.90,0.10)\rangle$ & $\langle 0.80,(0.81,0.13)\rangle$ & $\langle 0.75,(0.30,0.60)\rangle$ & $\langle 0.71,(0.50,0.40)\rangle$ \\
\hline$A_{5}$ & $\langle 0.80,(0.40,0.60)\rangle$ & $\langle 0.82,(0.80,0.10)\rangle$ & $\langle 0.75,(0.80,0.22)\rangle$ & $\langle 0.75,(0.60,0.40)\rangle$ & $\langle 0.73,(0.50,0.41)\rangle$ & $\langle 0.82,(0.90,0.13)\rangle$ \\
\hline
\end{tabular}

the confidence levels of the corresponding evaluation value, which was shown by using the decision matrices $Q_{1}, Q_{2}, Q_{3}$ (see Table 1,2,3). Further, we demand to select the best alternative and take full advantage of the novel method in the previous section to solve this problem. In the following, in order to show the MCGDM process based on the Cq-ROFHWA operator, we make use of the operator to solve the example, where $\mathrm{q}=3, \rho=3$.

Step 1. Take into account the six criteria are the benefit type, so it is not necessary to normalize with respect to the evaluation value.

Step 2. Compose all the q-rung orthopair fuzzy decision matrices $Q_{1}, Q_{2}, Q_{3}$ to the integrated decision matrix $\mathrm{Q}$ by using the Cq-ROFHWA operator, shown as Table 4.

Step 3. According to decision matrix $\mathrm{Q}$, the corresponding evaluation values $\phi_{i}(i=1, \ldots, m)$ of each alternative under various criteria are aggregated by using q-ROFHWA, shown as follows:

$$
\begin{aligned}
& \phi_{1}=(0.592,0.394), \phi_{2}=(0.557,0.349), \phi_{3}=(0.657,0.283), \\
& \phi_{4}=(0.571,0.424), \phi_{5}=(0.619,0.402) .
\end{aligned}
$$

Step 4. Based on the score function Eq. (2), we can obtain 


$$
\begin{aligned}
& S\left(\phi_{1}\right)=0.1463, S\left(\phi_{2}\right)=0.1303, S\left(\phi_{3}\right)=0.2609, \\
& S\left(\phi_{4}\right)=0.1099, S\left(\phi_{5}\right)=0.1722 .
\end{aligned}
$$

Step 5. According to the results of score function, we can choose the best alternative in descending order. Because

$$
A_{3}>A_{5}>A_{1}>A_{2}>A_{4} .
$$

So, the best robot company is $A_{3}$.

Table 4 q-rung orthopair fuzzy decision matrix $Q$

\begin{tabular}{ccccccc}
\hline & $G_{1}$ & $G_{2}$ & $G_{3}$ & $G_{4}$ & $G_{5}$ & $G_{6}$ \\
\hline$A_{1}$ & $(0.4696,0.4477)$ & $(0.5373,0.3718)$ & $(0.6633,0.4075)$ & $(0.7434,0.2532)$ & $(0.4796,0.5092)$ & $(0.599,0.4480)$ \\
\hline$A_{2}$ & $(0.5279,0.4369)$ & $(0.4723,0.3646)$ & $(0.7257,0.1980)$ & $(0.6037,0.2534)$ & $(0.3866,0.5814)$ & $(0.6049,0.3044)$ \\
\hline$A_{3}$ & $(0.6597,0.3238)$ & $(0.7293,0.2415)$ & $(0.5889,0.2043)$ & $(0.5793,0.3385)$ & $(0.7247,0.2670)$ & $(0.4982,0.4219)$ \\
\hline$A_{4}$ & $(0.5420,0.3950)$ & $(0.4558,0.4857)$ & $(0.8115,0.2233)$ & $(0.6172,0.3999)$ & $(0.3489,0.6443)$ & $(0.5308,0.3886)$ \\
\hline$A_{5}$ & $(0.5602,0.3693)$ & $(0.5783,0.3984)$ & $(0.7077,0.3178)$ & $(0.4875,0.5487)$ & $(0.6303,0.4374)$ & $(0.7530,0.3219)$ \\
\hline
\end{tabular}

5.2 Discussion about the influence of different aggregation operators and parameter values $\rho$ and $q$ on the results

In the following, we explore the influence of different AOs and parameter $\rho$ on the results, as shown in Figure 1,2,3,4,5,6 and Table 5,6,7,8. It is the ranking results obtained by adopting weighted, ordered weighted averaging operators, weighted, ordered weighted geometric operators respectively based on four operations of ATS when $q=3$. From Figure 1,2,3,4,5,6 and Table 5,6,7,8, we can summarize some important rules, shown as follows.

Table 5 Ranking results based on special ATS-Cq-ROFWA aggregation operators

\begin{tabular}{ccc}
\hline Methods & Score Values & Ranking \\
\hline Cq-ROFWA & $S\left(A_{1}\right)=0.2050, S\left(A_{2}\right)=0.1645, S\left(A_{3}\right)=0.3075, S\left(A_{4}\right)=0.1672, S\left(A_{5}\right)=0.2244$ & $A_{3}>A_{5}>A_{1}>A_{4}>A_{2}$ \\
Cq-ROFEWA & $S\left(A_{1}\right)=0.1690, S\left(A_{2}\right)=0.1433, S\left(A_{3}\right)=0.2784, S\left(A_{4}\right)=0.1323, S\left(A_{5}\right)=0.1922$ & $A_{3}>A_{5}>A_{1}>A_{2}>A_{4}$ \\
Cq-ROFHWA $(\rho=3)$ & $S\left(A_{1}\right)=0.1463, S\left(A_{2}\right)=0.1303, S\left(A_{3}\right)=0.2609, S\left(A_{4}\right)=0.1099, S\left(A_{5}\right)=0.1722$ & $A_{3}>A_{5}>A_{1}>A_{2}>A_{4}$ \\
Cq-ROFFWA $(\rho=2)$ & $S\left(A_{1}\right)=0.2307, S\left(A_{2}\right)=0.1911, S\left(A_{3}\right)=0.3567, S\left(A_{4}\right)=0.1861, S\left(A_{5}\right)=0.2565$ & $A_{3}>A_{5}>A_{1}>A_{2}>A_{4}$ \\
\hline
\end{tabular}

1. With the change of the parameter $\rho$, the algebraic operations and the Einstein operations can be served as specific forms of the Hamacher operations. Therefore, we can find that if weighted, ordered weighted geometric operators are used to calculate, the score values and the accuracy values decrease with the decrease of parameter value $\rho$. And whereas if weighted, ordered weighted averaging operators are used to calculate, the result is exactly the opposite. Furthermore, because 
Table 6 Ranking results based on special ATS-Cq-ROFWG aggregation operators

\begin{tabular}{clc}
\hline Methods & Score Values & Ranking \\
\hline Cq-ROFWG & $S\left(A_{1}\right)=0.1909, S\left(A_{2}\right)=0.1739, S\left(A_{3}\right)=0.3116, S\left(A_{4}\right)=0.1383, S\left(A_{5}\right)=0.1967$ & $A_{3}>A_{5}>A_{1}>A_{2}>A_{4}$ \\
Cq-ROFEWG & $S\left(A_{1}\right)=0.2287, S\left(A_{2}\right)=0.2013, S\left(A_{3}\right)=0.3458, S\left(A_{4}\right)=0.1715, S\left(A_{5}\right)=0.2296$ & $A_{3}>A_{5}>A_{1}>A_{2}>A_{4}$ \\
Cq-ROFHWG $(\rho=3)$ & $S\left(A_{1}\right)=0.2525, S\left(A_{2}\right)=0.2185, S\left(A_{3}\right)=0.3657, S\left(A_{4}\right)=0.1921, S\left(A_{5}\right)=0.2499$ & $A_{3}>A_{1}>A_{5}>A_{2}>A_{4}$ \\
Cq-ROFFWG $(\rho=2)$ & $S\left(A_{1}\right)=0.2549, S\left(A_{2}\right)=0.2299, S\left(A_{3}\right)=0.3957, S\left(A_{4}\right)=0.1896, S\left(A_{5}\right)=0.2602$ & $A_{3}>A_{5}>A_{1}>A_{2}>A_{4}$ \\
\hline
\end{tabular}

the algebraic operation is the specific form of the Frank operation when $\rho=1$, we can find that the ranking result of Frank operation and the Hamacher operation AOs change in the same way.

2. We know that each parameter value $\rho$ determines a kind of operation law based on ATS. So, by using different AOs when the parameter value $\rho$ is the same, it has been observed that the ranking results are slightly different. However, we can know that the best alternative is $A_{3}$.

3. As discussed above, the parameter value $\rho$ can be served as the expert's attitude, we can find that there is a negative correlation between the value of the parameter $\rho$ and the score value of the averaging operator. As the parameter value $\rho$ becomes larger, the pessimism of expert attitude will increase. On the contrary, when we use geometric operators to aggregate, we can find that there is a positive correlation between the value of the parameter $\rho$ and the score value. As the parameter value $\rho$ becomes larger, the optimism of expert attitude will increase. Therefore, the expert can choose the parameter value $\rho$ flexibly according to his attitude and the aggregation operator.

Table 7 Ranking results based on special ATS-Cq-ROFOWA aggregation operators

\begin{tabular}{clcl}
\hline Methods & Score Values & Ranking \\
\hline Cq-ROFOWA & $S\left(A_{1}\right)=0.2194, S\left(A_{2}\right)=0.1744, S\left(A_{3}\right)=0.3208, S\left(A_{4}\right)=0.1810, S\left(A_{5}\right)=0.2346$ & $A_{3}>A_{5}>A_{1}>A_{4}>A_{2}$ \\
Cq-ROFEOWA & $S\left(A_{1}\right)=0.1818, S\left(A_{2}\right)=0.1532, S\left(A_{3}\right)=0.2913, S\left(A_{4}\right)=0.1452, S\left(A_{5}\right)=0.2022$ & $A_{3}>A_{5}>A_{1}>A_{2}>A_{4}$ \\
Cq-ROFHOWA $(\rho=3)$ & $S\left(A_{1}\right)=0.1600, S\left(A_{2}\right)=0.1393, S\left(A_{3}\right)=0.2732, S\left(A_{4}\right)=0.1236, S\left(A_{5}\right)=0.1820$ & $A_{3}>A_{5}>A_{1}>A_{2}>A_{4}$ \\
Cq-ROFFOWA $(\rho=2)$ & $S\left(A_{1}\right)=0.2479, S\left(A_{2}\right)=0.2034, S\left(A_{3}\right)=0.3744, S\left(A_{4}\right)=0.2014, S\left(A_{5}\right)=0.2672$ & $A_{3}>A_{5}>A_{1}>A_{2}>A_{4}$ \\
\hline
\end{tabular}

Table 8 Ranking results based on special ATS-Cq-ROFOWG aggregation operators

\begin{tabular}{clcl}
\hline Methods & Score Values & Ranking \\
\hline Cq-ROFOWG & $S\left(A_{1}\right)=0.1993, S\left(A_{2}\right)=0.1814, S\left(A_{3}\right)=0.3187, S\left(A_{4}\right)=0.1450, S\left(A_{5}\right)=0.2042$ & $A_{3}>A_{5}>A_{1}>A_{2}>A_{4}$ \\
Cq-ROFEOWG & $S\left(A_{1}\right)=0.2372, S\left(A_{2}\right)=0.2094, S\left(A_{3}\right)=0.3520, S\left(A_{4}\right)=0.1783, S\left(A_{5}\right)=0.2370$ & $A_{3}>A_{1}>A_{5}>A_{2}>A_{4}$ \\
Cq-ROFHOWG $(\rho=3)$ & $S\left(A_{1}\right)=0.2595, S\left(A_{2}\right)=0.2252, S\left(A_{3}\right)=0.3735, S\left(A_{4}\right)=0.1988, S\left(A_{5}\right)=0.2581$ & $A_{3}>A_{1}>A_{5}>A_{2}>A_{4}$ \\
Cq-ROFFOWG $(\rho=2)$ & $S\left(A_{1}\right)=0.2649, S\left(A_{2}\right)=0.2394, S\left(A_{3}\right)=0.4063, S\left(A_{4}\right)=0.1968, S\left(A_{5}\right)=0.2695$ & $A_{3}>A_{5}>A_{1}>A_{2}>A_{4}$ \\
\hline
\end{tabular}


Furthermore, in order to analyse the influence of parameter value $q$ on the result, we list the results of different $q$ values based on the proposed Cq-ROFHWA operator in Table 9.

Table 9 Ranking results for different q based on Cq-ROFHWA

\begin{tabular}{ccc}
\hline Methods & Score Values & Ranking \\
\hline$q=2$ & $S\left(A_{1}\right)=0.1415, S\left(A_{2}\right)=0.1461, S\left(A_{3}\right)=0.3104, S\left(A_{4}\right)=0.0892, S\left(A_{5}\right)=0.1741$ & $A_{3}>A_{5}>A_{2}>A_{1}>A_{4}$ \\
$q=3$ & $S\left(A_{1}\right)=0.1463, S\left(A_{2}\right)=0.1303, S\left(A_{3}\right)=0.2609, S\left(A_{4}\right)=0.1099, S\left(A_{5}\right)=0.1722$ & $A_{3}>A_{5}>A_{1}>A_{2}>A_{4}$ \\
$q=5$ & $S\left(A_{1}\right)=0.1088, S\left(A_{2}\right)=0.0772, S\left(A_{3}\right)=0.1641, S\left(A_{4}\right)=0.0914, S\left(A_{5}\right)=0.1229$ & $A_{3}>A_{5}>A_{1}>A_{4}>A_{2}$ \\
$q=7$ & $S\left(A_{1}\right)=0.0757, S\left(A_{2}\right)=0.0445, S\left(A_{3}\right)=0.1062, S\left(A_{4}\right)=0.0647, S\left(A_{5}\right)=0.0827$ & $A_{3}>A_{5}>A_{1}>A_{4}>A_{2}$ \\
\hline
\end{tabular}

From Table 9, on the one hand, it has been observed that if $q=3$, the worst alternative is $A_{4}$. Where as if $q>3$ we get the different result that the worst alternative is $A_{2}$. In spite of this, the best alternative is $A_{3}$. On the other hand, we can find that the score value $S\left(A_{i}\right)$ decreases with the increase of parameter value $q$. When selecting the value of $q$, we should select the smallest $q$ value in the case of satisfying $\mu_{Q}^{q}+$ $v_{Q}^{q} \leq 1$. Such as, suppose the evaluation value is $(0.6,0.6)$, then we should choose the least value 2 that satisfies $0.6^{q}+0.6^{q} \leq 1$.

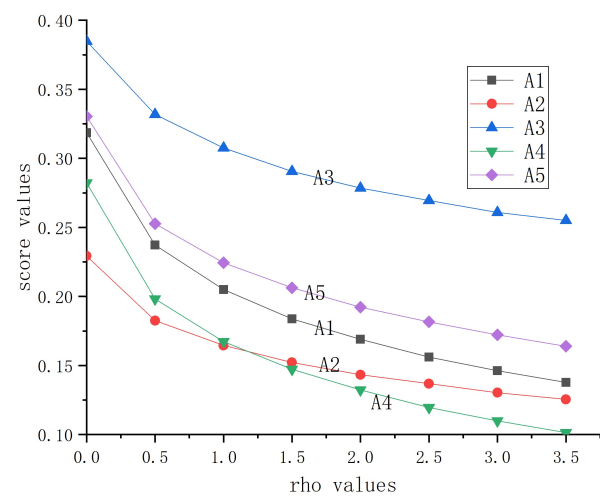

Fig. 1 Score values of the Cq-ROFHWA (where $q=3, \rho \in(0.0001,3.5)$ ).

From Figure 7,8, we can find that the score values and the accuracy values of ATSCq-ROFHOWG increase with the increase of parameter value $\rho$ and the decrease of parameter value $q$.

From Figure 9,10, we can find that the score values and the accuracy values of ATS-Cq-ROFHWA decrease with the increase of parameter value $\rho$ and $q$ value. 


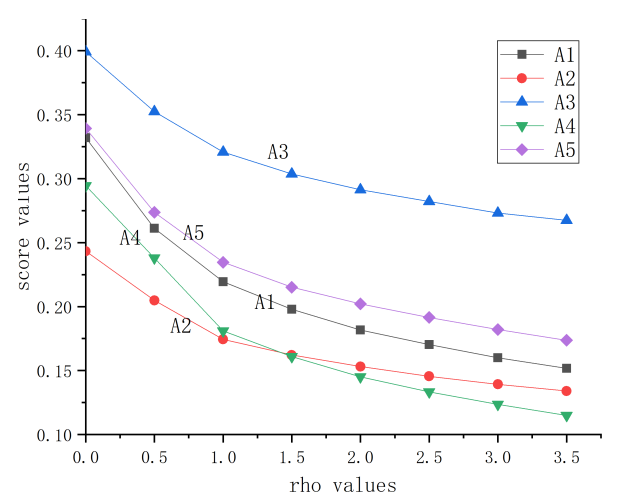

Fig. 2 Score values of the Cq-ROFHOWA (where $q=3, \rho \in(0.0001,3.5)$ ).

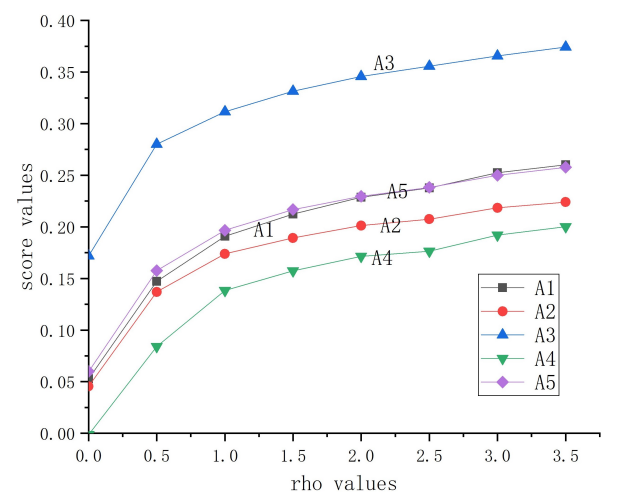

Fig. 3 Score values of the Cq-ROFHWG (where $q=3, \rho \in(0.0001,3.5)$ ).

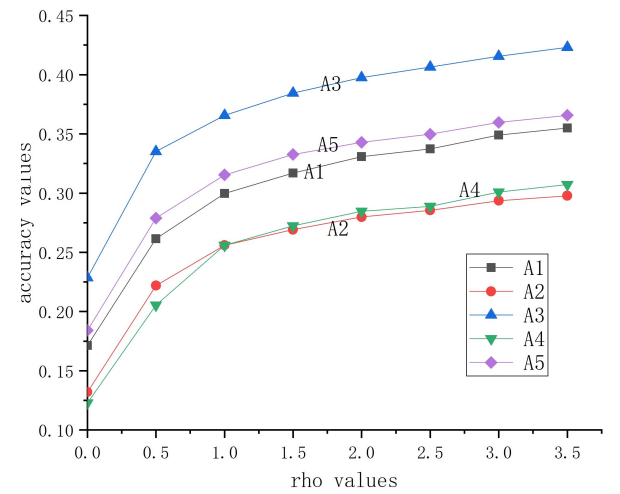

Fig. 4 Accuracy values of the Cq-ROFHWG (where $q=3, \rho \in(0.0001,3.5)$ ).

5.3 Comparing with the existing literature [29,23,24]

In the following, we demand to investigate the rationality and effectiveness of the proposed MCGDM method. To this end, three existing different methods were chosen 


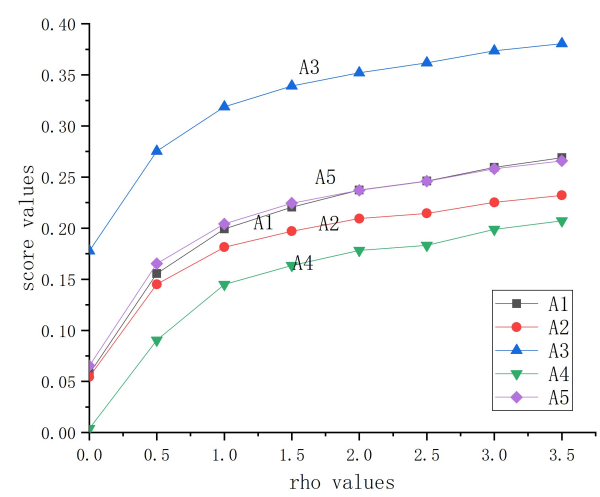

Fig. 5 Score values of the Cq-ROFHOWG (where $q=3, \rho \in(0.0001,3.5)$ ).

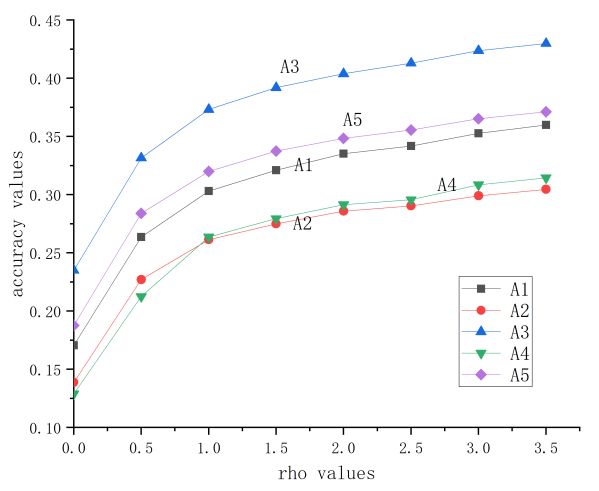

Fig. 6 Accuracy values of the Cq-ROFHOWG (where $q=3, \rho \in(0.0001,3.5)$ ).

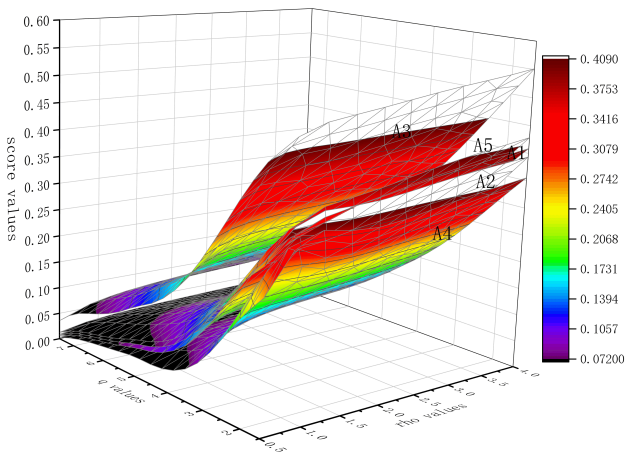

Fig. 7 Score values of the ATS-Cq-ROFHOWG (where $q=2,3,5,7, \rho \in(0.0001,3.5)$ ).

to handle this example and compare it with the ranking results. And we compare them 


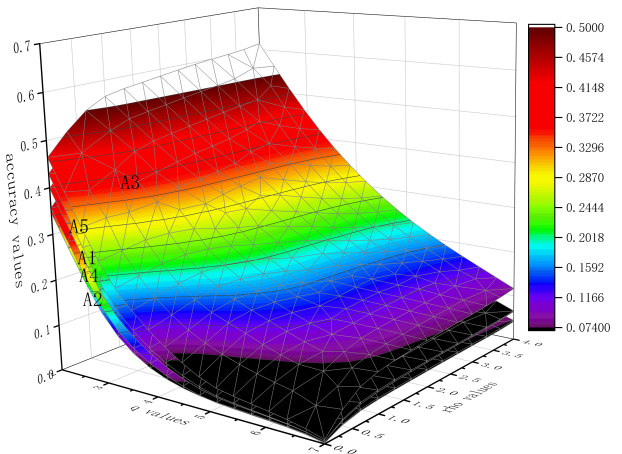

Fig. 8 Accuracy values of the ATS-Cq-ROFHOWG (where $q=2,3,5,7, \rho \in(0.0001,3.5)$ ).

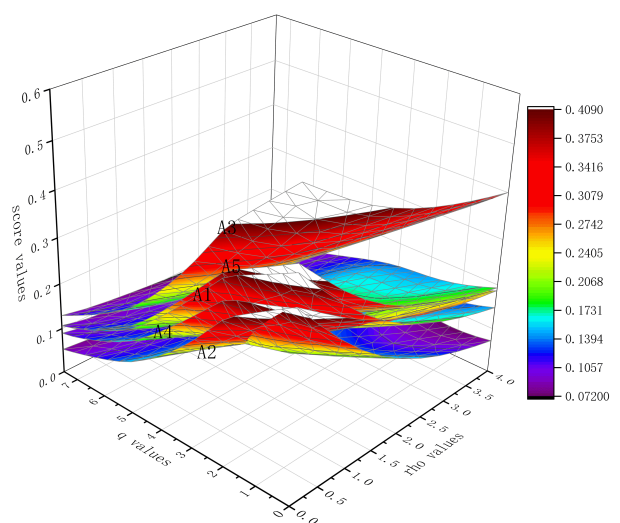

Fig. 9 Score values of the ATS-Cq-ROFHWA (where $q=2,3,5,7, \rho \in(0.0001,3.5)$ ).

Table 10 Ranking results for different MCGDM methods

\begin{tabular}{ccc}
\hline Methods & Score Values & Ranking \\
\hline $\begin{array}{c}\text { Peng } \text { et al.'s MCGDM Method [29] } \\
\text { (Based on the } q-\text { ROFWEA operator) } \\
\text { Liu and Chen's MCGDM Method [23] } \\
\text { (Based on the } I F W A H A \text { operator) }\end{array}$ & Cannot be aggregated & The ranking result cannot be obtained \\
$\begin{array}{c}\text { Liu and Liu's MCGDM Method [24] } \\
\text { (Based on the } q-R O F W B M \text { operator, } \\
\text { where } s=1, t=1 \text { ) }\end{array}$ & Cannot be aggregated & The ranking result cannot be obtained \\
The novel MCGDM Method & $S\left(A_{1}\right)=-0.181, S\left(A_{2}\right)=-0.223, S\left(A_{3}\right)=-0.129$, & \\
noved & $S\left(A_{4}\right)=-0.207, S\left(A_{5}\right)=-0.161$ & $A_{3}>A_{5}>A_{1}>A_{4}>A_{2}$ \\
(Based on the $C q-$ ROFHWA operator) & $S\left(A_{1}\right)=0.1463, S\left(A_{2}\right)=0.1303, S\left(A_{3}\right)=0.2609$, & \\
& $S\left(A_{4}\right)=0.1099, S\left(A_{5}\right)=0.1722$ & $A_{3}>A_{5}>A_{1}>A_{2}>A_{4}$ \\
\hline
\end{tabular}

with our proposed method concerning the results, which is shown in Table 10 (assume that the confidence level of all evaluation value is equal to $1, q=3$ ).

(1) Comparing Analysis with Peng et al.'s Mthod [29] Based on q-Rung Orthopair Fuzzy Weighted Exponential Aggregation (q-ROFWEA) Operator 


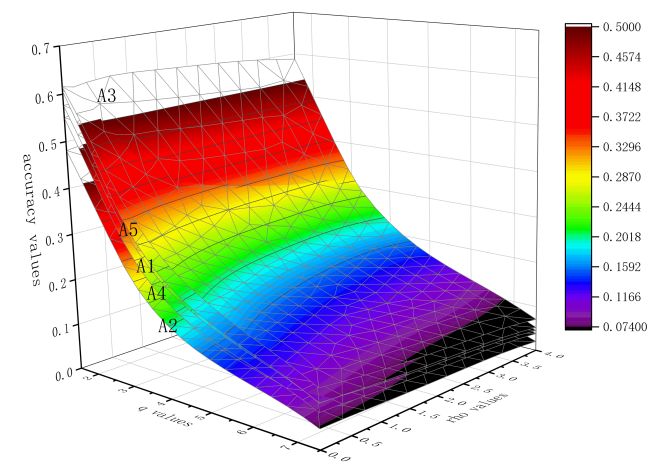

Fig. 10 Accuracy values of the ATS-Cq-ROFHWA (where $q=2,3,5,7, \rho \in(0.0001,3.5)$ ).

From [29], we can find that this method can't calculate the above example. In the following, we analyze the causes and defects.

1. In terms of information data, both of these methods are based on q-ROFNs, so their common advantage is that they can deal with the situation that the evaluation value satisfy $\mu_{Q}^{q}+v_{Q}^{q} \leq 1$.

2. In terms of operational laws, Peng et al.'s method [29] only considers the algebraic operation rules and cannot flexibly adjust the operation rules according to the attitude of the decision maker. Whereas the proposed method is based on ATS operation rules, thus the appropriate algorithm can be selected according to the attitude of the decision maker. Therefore, the proposed method is better than Peng et al.'s method [29], so it is more comprehensive when solving problems.

(2) Comparing Analysis with Liu and Chen's Method [23] Based on Intuitionistic Fuzzy Weighted Archimedean Heronian Aggregation (IFWAHA) Operator

We also can easily observe that this method can't handle this example. In the following, we compare and analyze the advantages and disadvantages of the two methods.

1. In terms of information data, It can be seen from $\langle 0.31,0.72\rangle$ in Table I that $0.31+0.72>1$, which does not satisfy $\mu_{Q}+v_{Q} \leq 1$. Therefore, Liu and Chen's method [23] can't deal with such data, whereas the method we proposed is based on the fact that $\left(\mu_{Q}, v_{Q}\right)$ is q-ROFNs, which can flexibly adjust the parameter value $q$ according to the requires of data in solving various uncertainty problems, so the proposed theory can be more widely applied and more effectively solve such problems.

2. In terms of operational laws, both of these methods are based on more general ATS operation, thus they are more versatile in solving uncertain problems.

3. In terms of the AOs, the proposed operator takes into account the confidence levels of evaluation value given by the decision maker, whereas Liu and Chen's theory takes into account the interrelationships between two evaluation attributes. 
Therefore, the two methods have different emphases in reducing decision deviation, and different applicable scenarios, with their own advantages and disadvantages.

(3) Comparing Analysis With Liu and Liu's Method [24] Based on q-Rung Orthopair Fuzzy Weighted Bonferroni Mean (q-ROFWBM) Operator

From [24], it has been observed that the ranking result is $A_{3}>A_{5}>A_{1}>A_{4}>A_{2}$, which is consistent with the proposed method. So, this illustrates the rationality of the proposed method. Then, compare the two methods and find out the merits of the proposed method.

1. In terms of information data, both of these methods are based on q-ROFNs to deal with uncertainty problem more comprehensively, and have the same advantages.

2. In terms of operational rules, Liu and Liu's method [24] only considers the calculation of AOs under the algebraic operation rules, so the ability to deal with problems is limited. However, The proposed method in this paper is based on ATS operations. Therefore, our method is more flexible and effective in selecting operational rules according to practical problems.

3. In terms of the AOs, Liu and Liu's method [24] aggregates data taking into account the interrelationships between evaluation attributes, whereas the proposed operator in this paper considers the confidence levels of evaluation value. Therefore, the two methods have different emphases in reducing decision deviation, and different applicable scenarios, with their own advantages and disadvantages. However, our method is too complicated to be suitable for big data decision problems. So the proposed method in this paper has obvious advantages in solving the MCGDM problem.

\subsection{Further comparing with the existing literature [21,22]}

We have previously confirmed the rationality and advantages of the proposed method by comparing it with the existing literature. However, by roughly consistent ranking results, we find that the advantages of the proposed method that takes into account the confidence levels cannot be reflected. Based on which, in order to demonstrate the advantages of the proposed method, we can obtain the ranking results by changing some data in the example and comparing them with the original data ranking results. Generally speaking, the evaluation value of alternative in decision data changes from large to small, which may have an impact on the ranking results. Therefore, we will gradually reduce the evaluation value of the best alternative $A_{3}$, and compare the processing ability of the proposed theory for extreme data. For example, we adapt the evaluation values $\tilde{\xi}_{34}^{2}$ and $\tilde{\xi}_{35}^{2}$ from $(\langle 0.89,(0.70,0.11)\rangle,\langle 0.85,(0.90,0.11)\rangle)$ to $(\langle 0.05,(0.01,0.90)\rangle,\langle 0.05,(0.01,0.90)\rangle)$ by gradually increasing NMD, and decreasing the confidence levels and MD. From common sense, we can foresee that the ranking result will change and the position of the best alternative $A_{3}$ will gradually move backward with the evaluation value decreases.

To further illustrate the superiority of the proposed method in this paper, we compare and analyze the proposed method with Liu and Wang's method [21] and Liu 
Table 11 Score values for different MCGDM methods

\begin{tabular}{cccc}
\hline$\left(\xi_{34}^{2}, \xi_{35}^{2}\right)$ & The proposed MCGDM method & Liu and Wang's MCGDM Method [21] & Liu et al.'s MCGDM Method [22] \\
\hline$(\langle 0.89,(0.70,0.11)\rangle$, & $S\left(A_{1}\right)=0.1463, S\left(A_{2}\right)=0.1303$, & $S\left(A_{1}\right)=0.2990, S\left(A_{2}\right)=0.2250$, & $S\left(A_{1}\right)=0.3228, S\left(A_{2}\right)=0.25165$, \\
$\langle 0.85,(0.90,0.11)\rangle)$ & $S\left(A_{3}\right)=0.2609, S\left(A_{4}\right)=0.1099$, & $S\left(A_{3}\right)=0.3740, S\left(A_{4}\right)=0.2520$, & $S\left(A_{3}\right)=0.4070, S\left(A_{4}\right)=0.25173$, \\
$\langle 0.5,(0.60,0.40)\rangle$ & $S\left(A_{5}\right)=0.1722$ & $S\left(A_{5}\right)=0.3001$ & $S\left(A_{5}\right)=0.3558$ \\
$\langle 0.4,(0.45,0.52)\rangle$ & $S\left(A_{3}\right)=0.1908$ & $S\left(A_{3}\right)=0.2711$ & $S\left(A_{3}\right)=0.3351$ \\
$\langle 0.25,(0.21,0.60)\rangle$ & $S\left(A_{3}\right)=0.1770$ & $S\left(A_{3}\right)=0.2512$ & $S\left(A_{3}\right)=0.3183$ \\
$\langle 0.05,(0.01,0.90)\rangle$ & $S\left(A_{3}\right)=0.1691$ & $S\left(A_{3}\right)=0.2437$ & $S\left(A_{3}\right)=0.3054$ \\
& $S\left(A_{3}\right)=0.1659$ & $S\left(A_{3}\right)=0.2424$ & $S\left(A_{3}\right)=0.3050$ \\
\hline
\end{tabular}

Table 12 Ranking results for different MCGDM methods

\begin{tabular}{cccc}
\hline$\left(\tilde{\xi}_{34}^{2}, \tilde{\xi}_{35}^{2}\right)$ & The proposed MCGDM method & Liu and Wang's MCGDM Method [21] & Liu et al.'s MCGDM Method [22] \\
\hline$(\langle 0.89,(0.70,0.11)\rangle$, & $A_{3}>A_{5}>A_{1}>A_{2}>A_{4}$ & $A_{3}>A_{5}>A_{1}>A_{4}>A_{2}$ & $A_{3}>A_{5}>A_{1}>A_{4}>A_{2}$ \\
$\langle 0.85,(0.90,0.11)\rangle)$ & $A_{3}>A_{5}>A_{1}>A_{2}>A_{4}$ & $A_{5}>A_{1}>A_{3}>A_{4}>A_{2}$ & $A_{5}>A_{3}>A_{1}>A_{4}>A_{2}$ \\
$\langle 0.5,(0.60,0.40)\rangle$ & $A_{3}>A_{5}>A_{1}>A_{2}>A_{4}$ & $A_{5}>A_{1}>A_{4}>A_{3}>A_{2}$ & $A_{5}>A_{1}>A_{3}>A_{4}>A_{2}$ \\
$\langle 0.4,(0.45,0.52)\rangle$ & $A_{5}>A_{3}>A_{1}>A_{2}>A_{4}$ & $A_{5}>A_{1}>A_{4}>A_{3}>A_{2}$ & $A_{5}>A_{1}>A_{3}>A_{4}>A_{2}$ \\
$\langle 0.25,(0.21,0.60)\rangle$ & $A_{5}>A_{3}>A_{1}>A_{2}>A_{4}$ & $A_{5}>A_{1}>A_{4}>A_{3}>A_{2}$ & $A_{5}>A_{1}>A_{3}>A_{4}>A_{2}$ \\
$\langle 0.05,(0.01,0.90)\rangle$ & & &
\end{tabular}

et al.'s method [22], where they aggregate the decision matrix using q-ROFWA and q-ROFPWMSM operators, respectively. While the proposed method aggregates the decision matrix using Cq-ROFWA operator. Obviously, Liu and Wang's method [21] simply aggregates data without considering the confidence levels of evaluation value given by the decision maker or the interrelationships between evaluation attributes. And Liu et al.'s method [22] considers the correlation between two or more evaluation attributes, whereas the proposed method considers the confidence levels of the evaluation value. The score values and the ranking results of these three methods are listed in Table 11 and Table 12 respectively (Suppose the confidence levels of the other two theoretical evaluation values is $1, q=3$ ).

From Table 11, we can observe that the score value of each method also decreases with the decrease of the evaluation value. From Table 12, we can observe that the change of the evaluation value has different effects on the ranking result of each method. When the evaluation value data changes, the results of Liu and Wang's method [21] and Liu et al.'s method [22] will be greatly affected. With the evaluation value becomes smaller and smaller, the best alternative will gradually move backward. But when the evaluation value of the proposed method changes from $(\langle 0.89,(0.70,0.11)\rangle,\langle 0.85,(0.90,0.11)\rangle)$ to $(\langle 0.4,(0.45,0.52)\rangle,\langle 0.4,(0.45,0.52)\rangle)$, the ranking result does not change. And with the evaluation value more and more small, the best alternative will only move backward one, the ranking result will be slightly affected.

We can draw some conclusions from the above analysis and compare the advantages of the proposed method with respect to other methods. As we all know, in the decision-making process, some decision makers will give unreasonable evaluation values for some reasons, which will lead to different evaluation results. Therefore, we need to eliminate the influence of these extreme data as much as possible, whereas the decision-making method determines how much impact these extreme data have 
on the ranking results. Obviously, because Liu and Wang's method [21] do not take into account the confidence levels of the evaluation value given by the decision maker or the interrelationships between evaluation attributes, it is most affected by unreasonable extreme data. And although Liu et al.'s method [22] considers the correlation between two or more evaluation attributes and reduces the influence of extreme data on decision results. In addition, Liu et al.'s method [22] is also vulnerable to the impact of such data from the perspective of ranking results and the processing ability of extreme data is not ideal. But, our operator considers the confidence levels of evaluation value and according to the ranking results, the alternative position remains unchanged as the evaluation value decreases. When the evaluation value decreases to $\langle 0.25,(0.21,0.60)\rangle$, the position of alternative $A_{3}$ remains the second place. Therefore, it is less affected by extreme data and relatively stable, and has strong processing ability for extreme data.

In the following, we compare the different aspects of the proposed method with other methods, as shown in Table 13, where 0 stands for "no" and 1 stands for "yes". Through comparative analysis, it has been concluded that the methods put forward by us and others are different in the aspect of reducing decision-making deviation. The methods of others emphasise that reduce the deviation between attributes, while we tend to reduce the deviation caused by expert decision-making. Two types of the methods have their own advantages, we should choose different theories according to different application scenarios, but the method we propose is less influenced by extreme data and is more suitable for dealing with uncertainty problems.

Table 13 Comparison of the characteristics of different MCGDM methods

\begin{tabular}{cccc}
\hline characteristics & Liu and Wang's Method [21] & Liu et al.'s Method [22] & The Proposed Method \\
\hline Whether the Confidence Levels is Considered & 0 & 0 & 1 \\
Whether the Interrelationship between Two or More & 0 & 1 & 0 \\
Attributes is Considered & 0 & 0 & 1 \\
Whether the Influences of Extreme Data can be Eliminated & 0 & 0 & 1 \\
Whether more Complex Information can be Processed & & 0 \\
\hline
\end{tabular}

In a word, our method optimizes the existing methods in literatures [48, 15, 8, 44] and expands the range of application, which makes decision-making more reasonable in terms of reducing the decision deviation of decision makers, or considering the attitude of experts by changing parameter value $\rho$.

\section{Conclusion}

The purpose of this paper is to propose a further novel method to rule out the decision deviation caused by the subjective factors such as the difference of knowledge background of decision maker by applying the ATS to Cq-ROFNs. Firstly, we have introduced a series of new AOs for Cq-ROFSs based on ATS, including the ATS-Cq-ROFWA, ATS-Cq-ROFWG, ATS-Cq-ROFOWA, ATS-Cq-ROFOWG operators. In addition, we have also discussed their corresponding properties. Moreover, 
each operator has four special forms, and with the parameters $\rho$ change, the AOs can be converted to each other. Furthermore, we proposed a novel approach to solve MCGDM problems by using the proposed AOs. Finally, we have given an example to illustrate the rationality of the proposed method, and compared it with the existing ones. In the future, we mainly focus on applying confidence levels to other fuzzy environments, such as hesitant Pythagorean fuzzy Maclaurin symmetric mean operators [12], hesitant fuzzy linguistic term sets [34], dual hesitant fuzzy soft sets [11], multi-granular fuzzy linguistic modelling [27], fuzzy multigranulation rough set [36], consensus building with individual consistency control [25], and using novel methods to solve practical problems to reduce the decision deviation.

Acknowledgements This work was supported in part by National Key Research and Development Program of China (2019QY(Y)0301, the National Natural Science Foundation of China under Grant Nos. 12061067, 62176033 and 61936001, and the Natural Science Foundation of Chongqing No. cstc2019jcyjcxttX0002.

\section{Conflict of interest}

The authors declare that they have no known competing financial interests or personal relationships that could have appeared to influence the work reported in this paper.

\section{Ethical approval}

This article does not contain any studies with human participants or animals performed by any of the authors.

\section{References}

1. K. Atanassov, Intuitionistic fuzzy sets, Fuzzy Sets and Systems, 20(1), 87-96 (1986).

2. K. Atanassov, Intuitionistic fuzzy sets: theory and applications, Heidelberg, Germany: Springer, 1999.

3. K. Bai, X. Zhu and J. Wang et al., Power partitioned Heronian mean operators for q-rung orthopair uncertain linguistic sets with their application to multiattribute group decision making, International Journal of Intelligent Systems, 1-35, (2019).

4. T. Chen and C. Li, Determining objective weights with intuitionistic fuzzy entropy measures: a comparative analysis, Information Science, 180(21), 4207-4222 (2010).

5. S. De, R. Biswas, and A. Roy, Some operations on intuitionistic fuzzy sets, Fuzzy Sets and Systems, 114(3), 477-484 (2000).

6. H. Garg, Distance and similarity measure for intuitionistic multiplicative preference relation and its application, International Journal for Uncertainty Quantification, 7(2), 117-133 (2017).

7. H. Garg, Novel neutrality operation-based Pythagorean fuzzy geometric aggregation operators for multiple attribute group decision analysis, International Journal of Intelligent Systems, 34(10), 2459-2489 (2019).

8. H. Garg, Confidence levels based Pythagorean fuzzy aggregation operators and its application to decision-making process, Computational and Mathematical Organization Theory, 23(4), 546571,(2017).

9. H. Garg, A new generalized Pythagorean fuzzy information aggregation using Einstein operations and its application to decision making, International Journal of Intelligent Systems, 31(9), 886-920 (2016). 
10. H. Garg, Generalized Pythagorean fuzzy geometric aggregation operators using Einstein t-norm and $\mathrm{t}$-conorm for multicriteria decision-making process, International Journal of Intelligent Systems, 32(6), 597-630 (2017)

11. H. Garg and R. Arora, Dual hesitant fuzzy soft aggregation operators and their application in decisionmaking, Cognitive Computing, 10(5), 769-789 (2018).

12. H. Garg, Hesitant Pythagorean fuzzy Maclaurin symmetric mean operators and its applications to multiattribute decision-making process, International Journal of Intelligent Systems 34(4) (2019) 601626.

13. B. Joshi, A. Singh and P. Bhatt et al., Interval valued q-rung orthopair fuzzy sets and their properties, Journal of Intelligent Fuzzy Systems, 35(5), 5225-5230 (2018).

14. R. Joshi and S. Kumar, Jensen-Tsalli's intuitionistic fuzzy divergence measure and its applications in medical analysis and pattern recognition, International Journal of Uncertainty, Fuzziness and Knowledge-Based Systems, 27(1), 145-169 (2019).

15. B. Joshi and A. Gegov, Confidence levels q-rung orthopair fuzzy aggregation operators and its applications to MCDM problems, International Journal of Intelligent Systems, 35(1), 125-149,(2020).

16. Y. Ju, A. Wang and J. Ma et al., Some q-rung orthopair fuzzy 2-tuple linguistic Muirhead mean aggregation operators and their applications to multiple-attribute group decision making, International Journal of Intelligent Systems, 1-30, (2019).

17. E. P. Klement and R. Mesiar, Eds., Logical, algebraic, analytic, and probabilistic aspects of triangular norms, New York, USA: Elsevier, 2005.

18. J. Li, X. Zhang, and Z. Gong, Aggregating of interval-valued intuitionistic uncertain linguistic variables based on Archimedean t-norm and it applications in group decision makings, Journal of Computational Analysis and Applications, 24(5), 874-885 (2018) .

19. Z. Liu, S. Wang, and P. Liu, Multiple attribute group decision making based on q-rung orthopair fuzzy Heronian mean operators, International Journal of Intelligent Systems, 33(12), 2341-2363 (2018).

20. X. Liu, Y. Xu and R. Montes et al., Analysis of self-confidence indices-based additive consistency for fuzzy preference relations with self-confidence and its application in group decision making, International Journal of Intelligent Systems, 34(5), 920-946 (2019).

21. P. Liu and P. Wang, Some q-rung orthopair fuzzy aggregation operators and their applications to multiple-attribute decision making, International Journal of Intelligent Systems, 33, 259-280 (2018).

22. P. Liu, S. Chen, and P. Wang, Multiple-attribute group decision-making based on q-rung orthopair fuzzy power maclaurin symmetric mean operators, IEEE Transactions on Systems Man CyberneticsSystems 1-16 (2018).

23. P. Liu and S. Chen, Group decision making based on Heronian aggregation operators of intuitionistic fuzzy numbers, IEEE Transactions on Systems Man Cybernetics-Systems, 47(9), 2514-2530 (2017).

24. P. Liu and J. Liu, Some q-rung orthopair fuzzy Bonferroni mean operators and their application to multi-attribute group decision making, International Journal of Intelligent Systems, 33(2), 315-347 (2018) .

25. C. Li, R. Rodriguez and L. Martinez et al., Consensus building with individual consistency control in group decision making, IEEE Transactions on Fuzzy Systems, 27(2), 319-332 (2019).

26. P. Liu and H. Gao, Some intuitionistic fuzzy power Bonferroni mean operators in the framework of Dempster-Shafer theory and their application to multicriteria decision making, Applied Soft Computing, 85, 139-177 (2019).

27. J. Morente-Molinera, X. Wu and A. Morfeq et al., A novel multi-criteria group decision-making method for heterogeneous and dynamic contexts using multi-granular fuzzy linguistic modelling and consensus measures, Information Fusion, 53, 240-250(2020).

28. Muhammad M, Humaira K, etc. T-Spherical Fuzzy Einstein Hybrid Aggregation Operators and Their Applications in Multi-Attribute Decision Making Problems, Symmetry 2020, 12, 365; doi: 10.3390/sym12030365.

29. X. Peng, J. Dai, and H. Garg, Exponential operation and aggregation operator for q-rung orthopair fuzzy set and their decision-making method with a new score function, International Journal of Intelligent Systems, 33(11), 2255-2282 (2018).

30. Q. Qin, F. Liang and L. Li et al., A TODIM-based multi-criteria group decision making with triangular intuitionistic fuzzy numbers, Applied Soft Computing, 55, 93-107 (2017).

31. M, Hamid MT, Afzal D, ect. Multi-criteria decision making in robotic agrifarming with q-rung orthopair m-polar fuzzy sets. PLoS ONE 16(2), 2021, e0246485. https://doi.org/10.1371/journal.pone.0246485. 
32. M Riaz, Hafiz MAF,Humaira Kalsoom, ect. A Robust q-Rung Orthopair Fuzzy Einstein Prioritized Aggregation Operators with Application towards MCGDM, Symmetry 2020, 12, 1058; doi:10.3390/sym12061058.

33. M Riaz, Ayesha R, Humaira K, ect. q-Rung Orthopair Fuzzy Geometric Aggregation Operators Based on Generalized and Group-Generalized Parameters with Application to Water Loss Management, Symmetry 2020, 12, 1236; doi:10.3390/sym12081236.

34. R. Rodriguez, L. Martinez, and F. Herrera, Hesitant fuzzy linguistic term sets for decision making, IEEE Transactions on Fuzzy Systems, 20(1), 109-119 (2012).

35. Z. Shi, Z. Gong, and Y. Shao, A novel similarity measure of intuitionistic fuzzy sets induced by triangular norm, In 2012 International Conference on Machine Learning and Cybernetics, 233-237 (2012).

36. B. Sun, X. Zhou, and N. Lin, Diversified binary relation-based fuzzy multigranulation rough set over two universes and application to multiple attribute group decision making, Information Fusion, 55, 91104 (2020).

37. C. Tan, B. Ma, and X. Chen, Intuitionistic fuzzy geometric aggregation operator based on fuzzy measure for multi-criteria group decision making, In 2009 Sixth International Conference on Fuzzy Systems and Knowledge Discovery, 4, 545-549 (2009).

38. Z. Wang and Y. Wang, Prospect theory-based group decision-making with stochastic uncertainty and 2-tuple aspirations under linguistic assessments, Information Fusion, 56, 81-92 (2020).

39. S. Wan, Power average operators of trapezoidal intuitionistic fuzzy numbers and application to multiattribute group decision making, Applied Mathematical Modelling, 37(6), 4112-4126 (2013).

40. S. Wan, Q. Wang, and J. Dong, The extended VIKOR method for multi-attribute group decision making with triangular intuitionistic fuzzy numbers, Knowledge-Based Systems, 52, 65-77 (2013).

41. Z. Xu, Intuitionistic fuzzy aggregation operators, IEEE Transactions on Fuzzy Systems, 15(6), 11791187 (2007)

42. Z. Xu and R. Yager, Some geometric aggregation operators based on intuitionistic fuzzy sets, International Journal of General Systems, 35(4), 417-433 (2006).

43. M. Xia, Z. Xu, and N. Chen, Induced aggregation under confidence levels, International Journal of Uncertainty Fuzziness and Knowledge-Based Systems, 19(2), 201-227 (2011).

44. M. Xia, Z. Xu, and B. Zhu, Some issues on intuitionistic fuzzy aggregation operators based on Archimedean t-conorm and t-norm, Knowledge-Based Systems, 31, 78-88 (2012).

45. R. Yager, Pythagorean fuzzy subsets, 2013 Joint IFSA World Congress and NAFIPS Annual Meeting (IFSA/NAFIPS), 57-61 (2013).

46. R. Yager, Pythagorean membership grades in multicriteria decision making, IEEE Transactions on Fuzzy Systems, 22(4), 958-965 (2014).

47. R. Yager, Generalized orthopair fuzzy sets, IEEE Transactions on Fuzzy Systems, 25(5), 1222-1230 (2017).

48. D. Yu, Intuitionistic fuzzy information aggregation under confidence levels, Applied Soft Computing, 19, 147-160 (2014).

49. L. Zadeh, Fuzzy sets, Information Technology and Control, 8(3), 338-353 (1965).

50. J. Zhan, H. Malik, and M. Akram, Novel decision-making algoritheorems based on intuitionistic fuzzy rough environment, International Journal of Machine Learning and Cybernetics, 10(6), 14591485 (2019)

51. J. Zhan and B. Sun, Covering-based intuitionistic fuzzy rough sets and applications in multi-attribute decision-making, Artificial Intelligence Review, 1-31 (2018). https://doi.org/10.1007/s10462-018-96747

52. K. Zhang, J. Zhan and W. Wu, On multi-criteria decision-making method based on a fuzzy rough set model with fuzzy $\alpha$-neighborhoods, IEEE Transactions on Fuzzy Systems, DOI: 10.1109/TFUZZ.2020.3001670.

53. W. Zhou, Z. Xu, and M. Chen, Preference relations based on hesitant-intuitionistic fuzzy information and their application in group decision making, Computers Industrial Engineering, 87, 163-175 (2015). 